\title{
Shoot Production and Mineral Nutrients of Five Microgreens as Affected by Hydroponic Substrate Type and Post-Emergent Fertilization
}

\author{
Tongyin Li *, Geoffrey T. Lalk, Jacob D. Arthur, Madeline H. Johnson and Guihong Bi \\ Department of Plant and Soil Sciences, Mississippi State University, Starkweli, MS 39762, USA; \\ gt131@msstate.edu (G.T.L.); jda360@msstate.edu (J.D.A.); mj1215@msstate.edu (M.H.J.); \\ gbi@pss.msstate.edu (G.B.) \\ * Correspondence: t1665@msstate.edu
}

check for

updates

Citation: Li, T.; Lalk, G.T.; Arthur, J.D.; Johnson, M.H.; Bi, G. Shoot Production and Mineral Nutrients of Five Microgreens as Affected by Hydroponic Substrate Type and Post-Emergent Fertilization. Horticulturae 2021, 7, 129. https:// doi.org/10.3390/horticulturae7060129

Academic Editor: Nazim Gruda

Received: 30 April 2021

Accepted: 31 May 2021

Published: 2 June 2021

Publisher's Note: MDPI stays neutral with regard to jurisdictional claims in published maps and institutional affiliations.

Copyright: (C) 2021 by the authors Licensee MDPI, Basel, Switzerland. This article is an open access article distributed under the terms and conditions of the Creative Commons Attribution (CC BY) license (https:/ / creativecommons.org/licenses/by/ $4.0 /)$.

\begin{abstract}
As a new specialty crop with high market value, microgreens are vegetable or herb seedlings consumed at a young age, 7-21 days after germination. They are known as functional food with high concentrations of mineral nutrients and health beneficial phytochemicals. Microgreen industry lacks standardized recommendations on cultural practices including species/variety selection, substrate choice, and fertilization management. This study evaluated shoot growth and mineral nutrient concentrations in five microgreens including four Brassica and one Raphanus microgreens as affected by four hydroponic pad types and post-emergent fertilization in two experiments in January and February 2020. The five microgreens varied in their shoot height, fresh, dry shoot weights, and mineral nutrient concentrations with radish producing the highest fresh and dry shoot weights. Radish had the highest nitrogen $(\mathrm{N})$ concentration and mustard had the highest phosphorus $(\mathrm{P})$ concentrations when grown with three hydroponic pads except for hemp mat. Hydroponic pad type altered fresh, dry shoot weights, and mineral nutrients in tested microgreens. Microgreens in hemp mat showed the highest shoot height, fresh, dry shoot weights, and potassium $(\mathrm{K})$ concentration, but the lowest $\mathrm{N}$ concentration in one or two experiments. One time post-emergent fertilization generally increased shoot height, fresh, dry shoot weights, and macronutrient concentrations in microgreens.
\end{abstract}

Keywords: shoot growth; yield; mineral nutrients; hydroponic pad; fertilization

\section{Introduction}

Microgreen is an emerging new specialty crop with high market value of USD 30-50 per pound (454 g) [1]. It is a collective term for vegetable, herb, grain, or flower seedlings consumed at a young stage [2-5]. Microgreens are harvested 7-21 days after germination with expanding cotyledons or first pair of true leaves [6]. A number of plant species in the families including Amaranthacea, Apiaceae, Asteraceae, Brassicaceae, Fabaceae, and Lamiaceae, have been grown as microgreens, among which Brassica crops are of the most grown species [7-11]. Microgreens are used by chef and consumers to enhance flavor, color, and texture in various foods and have become increasingly popular in recent years as consumer awareness of microgreen dietary value increases $[1,3,8]$. The high market value, increasing customer demand, and short production cycle had drawn interest among vegetable growers to produce microgreens [7].

Microgreens are reported to be four to six times nutrient denser than their mature counterparts [12-15]. They are considered functional food with high mineral nutrient concentrations and health-beneficial phytochemicals [15]. Xiao et al. [16] reported microgreen greens in the Brassicaceae family are most abundant in macronutrients of $\mathrm{K}$ and calcium (Ca) and micronutrients of iron (Fe) and zinc ( $\mathrm{Zn}$ ) after evaluating 30 varieties. They also contain high levels of antioxidant phytochemicals including ascorbic acid, carotenoids, glucosinolates, and polyphenols with substantial variations within and between species [6]. 
Microgreen lettuce (Lactuca sativa) have higher mineral concentrations including Ca, Magnesium $(\mathrm{Mg}), \mathrm{Fe}$, manganese (Mn), $\mathrm{Zn}$, selenium (Se), and molybdenum (Mo) and lower nitrate concentration than mature lettuce [10]. Basil (Ocimum basilicum) microgreens can be biofortified with Se to satisfy the human dietary need for this micronutrient [17]. There lacks standardized recommendations for cultural practices including species/variety and substrate choice, fertilization, and control of microenvironment in relation to shoot yield and nutrient compositions of microgreens $[4,9,18,19]$.

Fertilization was considered optional in microgreen production since seeds have stored nutrient for initial seedling growth. However, fertilizer application, premixed in growing substrate, or applied post-emergent was reported to result in fast growth and high yield of microgreen shoots [20]. The most economic fertilization treatments for arugula (Eruca vesicaria subsp. sativa) microgreens were daily post-emergent fertigation with $150 \mathrm{mg} \cdot \mathrm{L}^{-1} \mathrm{~N}$ or daily application of $75 \mathrm{mg} \cdot \mathrm{L}^{-1} \mathrm{~N}$ plus preincorporation of $1000 \mathrm{mg} \cdot \mathrm{L}^{-1} \mathrm{~N}$ [18]. Preharvest daily spray of $10 \mathrm{mM}$ calcium chloride solution on broccoli (Brassica oleracea var. italica) microgreens increased shoot biomass, Ca concentration, and improved visual quality during storage. Plant species/variety, slow versus fast growing, may differ in their requirements for fertilization [1]. With an increasing number of species and varieties grown as microgreens, crop-specific fertilizer requirements with respect to microshoot yield and mineral nutrients largely remain unclear.

Microgreens can be produced with peat-based potting mix or hydroponic pads that are made from synthetic or recycled fibrous materials [7]. A variety of hydroponic pads including biostrate (made from felt fiber), jute mat, and hemp mat are commercially available, some of which are compostable and can serve as a sustainable alternative to a peat-based substrate [21,22]. Physical and chemical properties including container capacity, air filled porosity, bulk density, $\mathrm{pH}$, and electrical conductivity (EC) vary among substrate types and affect growth of microgreen crops [21]. Efficacy of using hydroponic mats to grow microgreens is species or variety dependent. Hydroponic pads made from textile fibers and jute-kenaf fibers were reported to produce similar fresh shoot yield of rapini (Brassica rapa L.) microgreens [21]. Hydroponic substrate type also affects nutritional facts of microgreens and food safety in microgreen production due to the humid conditions and warm temperatures. The latter has become one of the most important concerns in microgreen production [7], where hydroponic mat type was found to affect microbial populations on microgreens [21].

The objective of this study was to investigate shoot production and mineral nutrients of five microgreens grown with four types of hydroponic pads as affected by post-emergent fertilization.

\section{Materials and Methods}

\subsection{Plant Materials and Cultivation}

Shoot growth and mineral nutrient concentrations of five microgreens were evaluated (Table 1). Microgreen seeds of selected species were purchased from the True Leaf Market (Salt Lake City, UT, USA). Seed sowing rate for each microgreen was determined by supplier recommendation and summarized in Table 1. Hundred-seed weight of each microgreen was measured with three replications. This study was conducted in a greenhouse at Mississippi State University, USA. $\left(33.4552^{\circ} \mathrm{N}, 88.7944^{\circ} \mathrm{W}\right)$ and included two experiments with the first conducted on 29 January 2020 and then repeated on 25 February 2020. The temperature in the greenhouse was set at $25^{\circ} \mathrm{C}$ with natural light.

Each microgreen was grown with four types of hydroponic pad including biostrate, hemp mat, micro-mats, and jute mat (Table 2). Each hydroponic pad was precut or manually cut into the size of approximately $25 \mathrm{~cm}$ by $25 \mathrm{~cm}$ to fit the bottom of the growing tray. Black plastic trays without drainage holes (width $25.72 \mathrm{~cm}$, length $25.72 \mathrm{~cm}$, depth $6.03 \mathrm{~cm}$; T.O. Plastics, Clearwater, MN, USA) were used to grow microgreens in this study. Hydroponic pads were then hydrated by soaking them into tap water, drained with excessive water, and placed into each growing tray. Seeds of appropriate weight were measured and manually 
sown onto the hydrated hydroponic pads, and covered with another black tray to provide a dark environment and maintain moisture during germination. Microgreen seeds were misted with a spray bottle every $12 \mathrm{~h}$ to moisturize the seeds and substrate, when the cover tray was removed shortly and put back on after misting. Four days after sowing, the cover tray was turned upside down with the bottom being placed on top of microgreen shoots for another 24-48 h. This practice was to provide some resistance and encourage microshoot elongation as recommended by the seed supplier. The cover tray was then removed at approximately 7 days after planting (DAP).

Table 1. Common name, scientific name, seeding rate, 100 seed weight, and harvest date of five microgreens.

\begin{tabular}{|c|c|c|c|c|c|}
\hline $\begin{array}{l}\text { Common } \\
\text { Name }\end{array}$ & Scientific Name & $\begin{array}{l}\text { Seeding Rate } \\
\left(\mathrm{g} \cdot \mathrm{m}^{-2}\right)\end{array}$ & $\begin{array}{l}100 \text { Seed Wt. } \\
(\mathrm{g})\end{array}$ & $\begin{array}{l}\text { Harvest Date } \\
\quad \text { (DAP) }\end{array}$ & Harvest Stage ${ }^{1}$ \\
\hline Broccoli & $\begin{array}{c}\text { Brassica oleracea var. italica cv. } \\
\text { 'Waltham' }\end{array}$ & 98.3 & $0.44 \pm 0.015$ & $12-14$ & Stage 1 \\
\hline Cabbage & $\begin{array}{c}\text { Brassica oleracea var. capitata cv. 'Red } \\
\text { Acre' }\end{array}$ & 83.1 & $0.44 \pm 0.016$ & $14-15$ & Stage 1 \\
\hline Kale & $\begin{array}{c}\text { Brassica napus var. pabularia cv. 'Red } \\
\text { Russian' }\end{array}$ & 75.6 & $0.23 \pm 0.008$ & $14-16$ & Stage 1 \\
\hline Mustard & Brassica juncea cv. 'Red Garnet' & 60.5 & $0.18 \pm 0.003$ & $15-16$ & $\begin{array}{c}\text { Mixed with } \\
70-80 \% \text { stage } 1 \\
\text { shoots }\end{array}$ \\
\hline Radish & Raphanus sativus cv. 'Rambo' & 189.0 & $1.10 \pm 0.03$ & $12-14$ & Stage 1 \\
\hline
\end{tabular}

${ }^{1}$ Microgreens were harvested with the expanding cotyledons (microgreen stage 1) or with the first pair of true leaves (microgreen stage 2).

Table 2. Product name, constructed material, supplier, and manufacturer information of the four types of hydroponic pads used in this study.

\begin{tabular}{|c|c|c|c|}
\hline Hydroponic Pad Product & Materials & Supplier & Manufacturer \\
\hline Biostrate $^{\circledR}$ & Felt fiber & True Leaf Market & Quick Plug, South Portland, ME, USA \\
\hline Hemp mat & Hemp fiber & Amazon & Terrafibre, Drayton Valley, Canada \\
\hline Jute mat & Jute fiber & True Leaf Market & Handy Pantry, Salt Lake City, UT, USA \\
\hline Micro-Mats & Wood fiber & True Leaf Market & Handy Pantry, Salt Lake City, UT, USA \\
\hline
\end{tabular}

After covers were removed, half of the trays from each microgreen were fertigated once with $120 \mathrm{~mL}$ of water-soluble fertilizer 20N-8.7P-16.6K (Peters ${ }^{\circledR}$ Professional 20-2020 General Purpose, also containing (wt/wt) $0.05 \% \mathrm{Mg}, 0.05 \% \mathrm{Fe}, 0.025 \% \mathrm{Mn}, 0.013 \%$ boron (B), $0.013 \%$ copper (Cu), $0.005 \%$ Mo, and $0.025 \%$ Zn; ICL Specialty Fertilizers, TelAviv, Israel) at a rate of $100 \mathrm{mg} \cdot \mathrm{L}^{-1} \mathrm{~N}$. The fertilizer solution had a $\mathrm{pH}$ of 6.56 and EC of $0.41 \mathrm{mS} \cdot \mathrm{cm}^{-1}$. As a control to the fertilization treatment, the other half of trays were irrigated with the same volume of water ( $\mathrm{pH} 7.54$; EC $\left.0.15 \mathrm{mS} \cdot \mathrm{cm}^{-1}\right)$.

\subsection{Data Collection and Shoot Harvest}

Plant height was measured in each tray before shoot harvest from the substrate surface to the highest point of shoot growth. Microgreen shoot in each tray were carefully harvested above the substrate surface, with the expanding cotyledons (microgreen stage 1) or with the first pair of true leaves (microgreen stage 2) as described by Waterland et al. [5]. Fresh shoot weight of microgreens harvested from each tray was measured. Freshly harvested microgreen shoots were then oven dried at $60{ }^{\circ} \mathrm{C}$ until constant weight and measured for dry shoot weight (DW). Dry weight percentage (\%) was also determined for each tray.

\subsection{Mineral Nutrient Analyses}

Dry microgreen samples were ground to pass a 1-mm sieve with a grinder (Wiley mini mill, Thomas Scientific, Swedesboro, NJ, USA) for mineral nutrient analyses. Combustion analysis was used for the determination of total $\mathrm{N}$ concentration with $0.25 \mathrm{~g}$ of dry tissue 
using an elemental analyzer (vario MAX cube; Elementar Americas Inc., Long Island, NY, USA). A dry tissue sample of $0.5 \mathrm{~g}$ was digested with $1 \mathrm{~mL}$ of $6 \mathrm{M}$ hydrochloric acid $(\mathrm{HCl})$ and $50 \mathrm{~mL}$ of $0.05 \mathrm{M} \mathrm{HCl}$ for the concentrations of $\mathrm{P}, \mathrm{K}, \mathrm{Ca}, \mathrm{Mg}, \mathrm{Cu}, \mathrm{Fe}, \mathrm{Mn}, \mathrm{Zn}$, and B using inductively coupled plasma optical emission spectrometry (SPECTROBLUE; SPECTRO Analytical Instruments, Kleve, Germany). Microgreen samples were tested at the Mississippi State University Extension Service Soil Testing Laboratory. Concentrations of macronutrients $\left(\mathrm{mg} \cdot \mathrm{g}^{-1}\right)$ and micronutrients $\left(\mu \mathrm{g} \cdot \mathrm{g}^{-1}\right)$ in microgreens were presented on a dry weight basis.

\subsection{Experimental Design and Statistical Analyses}

This experiment was conducted in a randomized complete block design with a factorial arrangement of treatments. Microgreens (5 species), hydroponic pad (4 types), and fertilization (fertilizer or not) were the three main factors contributing to 40 treatment combinations. Each treatment combination had five replications with an individual growing tray as the experimental unit. Significance of any main effect or the interaction among main factors were determined by analysis of variance (ANOVA) using PROC GLMMIX procedure of SAS (version 9.4; SAS Institute, Cary, NC, USA). Where indicated by ANOVA, means were separated by Tukey's honest significant difference (HSD) at $\alpha \leq 0.05$. Data from the two experiments were compared as repeated measures. All statistical analyses were performed using SAS.

\section{Results}

The experiment date affected all measured dependent variables in this study, showing varying trends between the January and February experiments (data not shown). Therefore, data from the two experiments were presented separately. There was no three-way interaction among microgreen species, hydroponic substrate type, and fertilization treatment for any measured variable.

\subsection{Shoot Height}

Shoot height was affected by the interaction between microgreen species and hydroponic pad type in both experiments (Tables 3 and 4). Hemp resulted in the largest shoot height within each microgreen, with biostrate and jute mat resulting in similar shoot height in four microgreens next to hemp in January and February 2020. Micro-mats resulted in the lowest height in broccoli, kale, and radish in both experiments. Shoot height of radish and broccoli were higher than cabbage, kale, or mustard grown with any substrate type in both experiments. One time post-emergent fertilization increased shoot height by $3.2 \%$ and $2.5 \%$ in January and February 2020, respectively compared to the no fertilizer treatment (Table 5).

\subsection{Fresh and Dry Shoot Weights}

Microgreen shoots were harvested with expanding cotyledons or the first pair of true leaves with varying days ranging from 12 to 16 DAP (Table 1, Figure S1). Fresh, dry shoot weights, and dry weight percentage were affected by the interaction between microgreen species and hydroponic pad type in January 2020 (Table 3). Hemp mat resulted in higher fresh and dry shoot weights in each microgreen than other substrates in January (Table 3). Micro-Mats resulted in the lowest fresh and dry shoot weights in broccoli, kale, and radish. Among the five microgreens, radish produced the highest fresh and dry shoot weights when grown with each substrate type, with broccoli being the second highest, higher than kale, cabbage, or mustard. Dry weight percentage ranged from $5.48 \%$ in kale grown with hemp to $9.21 \%$ in mustard grown with Micro-Mats. The comparable highest dry weight percentages were found in cabbage, kale, and mustard grown with Micro-Mats and cabbage grown with biostrate. Hemp resulted in the lowest dry weight percentages in cabbage (5.81\%), kale (5.48\%), and mustard (6.78\%) among the four substrate types. 
Fresh shoot weight and dry weight percentage were also affected by the interaction between species and fertilization treatment in January 2020, when one time post-emergent fertilization increased fresh shoot weight and decreased dry weight percentage in kale and radish, but resulted in similar values of both variables in broccoli, cabbage, and radish (Table 6). One time fertilization also increased overall dry shoot weight by $2.7 \%$ in the February experiment (Table 5).

Table 3. Shoot height, fresh shoot weight, dry shoot weight, and dry weight percentage of five microgreens affected by the interaction between species and hydroponic pad type in January 2020.

January 2020

\begin{tabular}{|c|c|c|c|c|c|}
\hline Microgreens & Hydroponic Pad & $\begin{array}{c}\text { Shoot Height }{ }^{1,2} \\
(\mathrm{~cm})\end{array}$ & $\begin{array}{l}\text { Fresh Shoot Weight } \\
\qquad\left(\mathrm{g} \cdot \mathrm{m}^{-2}\right)\end{array}$ & $\begin{array}{l}\text { Dry Shoot Weight } \\
\left(\mathrm{g} \cdot \mathrm{m}^{-2}\right)\end{array}$ & $\begin{array}{c}\text { Dry Weight Percentage } \\
(\%)\end{array}$ \\
\hline \multirow{4}{*}{ Broccoli } & Biostrate & $6.69 \mathrm{~cd}$ & 1322 ef & 89.1 ef & $6.76 \mathrm{de}$ \\
\hline & Hemp & $8.32 \mathrm{~b}$ & 1843 c & $109.8 \mathrm{~d}$ & $6.04 \mathrm{efg}$ \\
\hline & Jute & $6.87 \mathrm{c}$ & 1512 de & $100.1 \mathrm{de}$ & $6.63 \mathrm{def}$ \\
\hline & Micro-Mats & 5.78 ef & $1136 \mathrm{fg}$ & $76.7 \mathrm{gh}$ & $6.77 \mathrm{de}$ \\
\hline \multirow{4}{*}{ Cabbage } & Biostrate & 4.85 ghi & $533 \mathrm{j}$ & 46.01 & $8.78 \mathrm{ab}$ \\
\hline & Hemp & $6.71 \mathrm{~cd}$ & $1462 \mathrm{de}$ & $84.9 \mathrm{fg}$ & $5.81 \mathrm{fg}$ \\
\hline & Jute & $5.3 \mathrm{fgh}$ & 817 hi & $64.7 \mathrm{ij}$ & $7.92 \mathrm{bc}$ \\
\hline & Micro-Mats & $4.61 \mathrm{hij}$ & $539 \mathrm{j}$ & $48.5 \mathrm{kl}$ & $9.10 \mathrm{a}$ \\
\hline \multirow{4}{*}{ Kale } & Biostrate & $5.24 \mathrm{fgh}$ & $1003 \mathrm{gh}$ & 70.7 hi & $7.23 \mathrm{~cd}$ \\
\hline & Hemp & $6.9 \mathrm{c}$ & 1487 de & $81.5 \mathrm{fgh}$ & $5.48 \mathrm{~g}$ \\
\hline & Jute & $5.35 \mathrm{efg}$ & $1000 \mathrm{gh}$ & $63.6 \mathrm{ij}$ & $6.55 \mathrm{def}$ \\
\hline & Micro-Mats & $3.92 \mathrm{jk}$ & $656 \mathrm{ij}$ & $58.2 \mathrm{jk}$ & $9.06 \mathrm{a}$ \\
\hline \multirow{4}{*}{ Mustard } & Biostrate & $4.28 \mathrm{ijk}$ & $533 j$ & 42.41 & $7.99 \mathrm{bc}$ \\
\hline & Hemp & $6.05 \mathrm{de}$ & 941 gh & $63.8 \mathrm{ij}$ & $6.78 \mathrm{de}$ \\
\hline & Jute & $4.34 \mathrm{ij}$ & $612 \mathrm{ij}$ & $48.1 \mathrm{kl}$ & $7.87 \mathrm{bc}$ \\
\hline & Micro-Mats & $3.58 \mathrm{k}$ & $468 \mathrm{j}$ & 43.01 & $9.21 \mathrm{a}$ \\
\hline \multirow{4}{*}{ Radish } & Biostrate & $7.25 \mathrm{~b}$ & $2278 \mathrm{~b}$ & $152.2 \mathrm{~b}$ & $6.77 \mathrm{de}$ \\
\hline & Hemp & $9.71 \mathrm{a}$ & $3158 \mathrm{a}$ & $186.4 \mathrm{a}$ & $5.92 \mathrm{efg}$ \\
\hline & Jute & $8 \mathrm{~b}$ & $2414 \mathrm{~b}$ & $160.8 \mathrm{~b}$ & $6.69 \mathrm{def}$ \\
\hline & Micro-Mats & $6.01 \mathrm{de}$ & $1676 \mathrm{~cd}$ & $122.9 \mathrm{c}$ & $7.46 \mathrm{~cd}$ \\
\hline \multirow[t]{3}{*}{$p$-value } & Species & $<0.0001$ & $<0.0001$ & $<0.0001$ & $<0.0001$ \\
\hline & Substrate & $<0.0001$ & $<0.0001$ & $<0.0001$ & $<0.0001$ \\
\hline & $\begin{array}{l}\text { Species* } \\
\text { Substrate }^{3}\end{array}$ & $<0.0001$ & $<0.0001$ & $<0.0001$ & $<0.0001$ \\
\hline
\end{tabular}

${ }^{1}$ Different lower case letters suggest significant difference among means within a column indicated by the Tukey's HSD test at $p \leq 0.05$.

${ }^{2}$ Each mean was obtained by averaging data from both the fertilized and no-fertilizer treatments. ${ }^{3}$ Species * Substrate suggests interaction

between species and substrate type.

Table 4. Shoot height, fresh shoot weight, dry shoot weight, and dry weight percentage of five microgreens affected by the interaction between species and hydroponic pad type or the main effect of specie in February 2020.

February 2020

\begin{tabular}{|c|c|c|c|c|c|}
\hline Microgreens & Hydroponic Pad & $\begin{array}{l}\text { Shoot Height } 1,2 \\
(\mathrm{~cm})\end{array}$ & $\begin{array}{l}\text { Fresh Shoot Weight } \\
\qquad\left(\mathrm{g} \cdot \mathrm{m}^{-2}\right)\end{array}$ & $\begin{array}{c}\text { Dry Shoot Weight } \\
\left(\mathrm{g} \cdot \mathrm{m}^{-2}\right)\end{array}$ & $\begin{array}{c}\text { Dry Weight Percentage } \\
(\%)\end{array}$ \\
\hline Broccoli & $\begin{array}{l}\text { Biostrate } \\
\text { Hemp } \\
\text { Jute } \\
\text { Micro-Mats }\end{array}$ & $\begin{array}{c}7.08 \mathrm{c} \\
8.31 \mathrm{a} \\
7.45 \mathrm{~b} \\
6.55 \mathrm{~cd}\end{array}$ & $1289 \mathrm{~b}$ & $84.9 \mathrm{~b}$ & $6.68 \mathrm{a}$ \\
\hline Cabbage & $\begin{array}{l}\text { Biostrate } \\
\text { Hemp } \\
\text { Jute } \\
\text { Micro-Mats }\end{array}$ & $\begin{array}{l}4.59 \mathrm{f}-\mathrm{i} \\
5.79 \mathrm{e} \\
5.01 \mathrm{f} \\
4.18 \mathrm{hi}\end{array}$ & $968 c$ & $67.8 \mathrm{c}$ & $7.20 \mathrm{a}$ \\
\hline
\end{tabular}


Table 4. Cont.

\begin{tabular}{|c|c|c|c|c|c|}
\hline \multirow[b]{2}{*}{ Microgreens } & \multirow[b]{2}{*}{ Hydroponic Pad } & \multicolumn{4}{|c|}{ February 2020} \\
\hline & & $\begin{array}{l}\text { Shoot Height }{ }^{1,2} \\
(\mathrm{~cm})\end{array}$ & $\begin{array}{l}\text { Fresh Shoot Weight } \\
\qquad\left(\mathrm{g} \cdot \mathrm{m}^{-2}\right)\end{array}$ & $\begin{array}{c}\text { Dry Shoot Weight } \\
\qquad\left(\mathrm{g} \cdot \mathrm{m}^{-2}\right)\end{array}$ & $\begin{array}{c}\text { Dry Weight Percentage } \\
(\%)\end{array}$ \\
\hline \multirow{4}{*}{ Kale } & Biostrate & $4.75 \mathrm{fgh}$ & \multirow{4}{*}{$929 \mathrm{c}$} & \multirow{4}{*}{$71.2 \mathrm{c}$} & \multirow{4}{*}{$7.90 \mathrm{a}$} \\
\hline & Hemp & $6.53 \mathrm{~cd}$ & & & \\
\hline & Jute & $4.93 \mathrm{f}$ & & & \\
\hline & Micro-Mats & $3.96 \mathrm{i}$ & & & \\
\hline \multirow{4}{*}{ Mustard } & Biostrate & $4.26 \mathrm{fhi}$ & \multirow{4}{*}{$626 \mathrm{~d}$} & \multirow{4}{*}{$46.9 \mathrm{~d}$} & \multirow{4}{*}{$7.71 \mathrm{a}$} \\
\hline & Hemp & $6.25 \mathrm{de}$ & & & \\
\hline & Jute & $4.83 \mathrm{f}$ & & & \\
\hline & Micro-Mats & 4.25 ghi & & & \\
\hline \multirow{4}{*}{ Radish } & Biostrate & $7.24 \mathrm{~b}$ & \multirow{4}{*}{$2325 \mathrm{a}$} & \multirow{4}{*}{156.3 a } & \multirow{4}{*}{$7.97 \mathrm{a}$} \\
\hline & Hemp & $8.92 \mathrm{a}$ & & & \\
\hline & Jute & $7.24 \mathrm{~b}$ & & & \\
\hline & Micro-Mats & $6.3 \mathrm{de}$ & & & \\
\hline \multirow[t]{3}{*}{$p$-value } & Species & $<0.0001$ & $<0.0001$ & $<0.0001$ & 0.58 \\
\hline & Substrate & $<0.0001$ & $<0.0001$ & $<0.0001$ & 0.24 \\
\hline & $\begin{array}{l}\text { Species* } \\
\text { Substrate }^{3}\end{array}$ & 0.0002 & 0.81 & 0.18 & 0.39 \\
\hline
\end{tabular}

${ }^{1}$ Different lower case letters suggest significant difference among means within a column indicated by the Tukey's HSD test at $p \leq 0.05$.

${ }^{2}$ Means for shoot height were obtained by averaging data from both the fertilized and no-fertilizer treatments; means of each microgreen species for fresh, dry shoot weights, and dry weight percentage were obtained by averaging data from both fertilization treatments and all four substrate types. ${ }^{3}$ Species * Substrate suggests interaction between species and substrate type.

Table 5. Microgreen shoot growth and mineral nutrient concentrations affected by the main effect of post-emergent fertilization.

\begin{tabular}{|c|c|c|c|c|c|c|c|}
\hline \multirow[b]{2}{*}{ Fertilization } & \multicolumn{4}{|c|}{ January 2020} & \multicolumn{3}{|c|}{ February 2020} \\
\hline & $\begin{array}{l}\text { Shoot Height }{ }^{1} \\
\text { (cm) }\end{array}$ & $\begin{array}{c}\text { Dry Shoot Weight } \\
\left(\mathrm{g} \cdot \mathrm{m}^{-2}\right)\end{array}$ & $\begin{array}{c}\mathrm{Mg}^{2} \\
\left(\mathrm{mg} \cdot \mathrm{g}^{-1}\right)\end{array}$ & $\begin{array}{c}\mathrm{S} \\
\left(\mathrm{mg} \cdot \mathrm{g}^{-1}\right)\end{array}$ & $\begin{array}{l}\text { Shoot Height } \\
\text { (cm) }\end{array}$ & $\begin{array}{c}\mathrm{N} \\
\left(\mathrm{mg} \cdot \mathrm{g}^{-1}\right)\end{array}$ & $\begin{array}{c}\mathrm{P} \\
\left(\mathrm{mg} \cdot \mathrm{g}^{-1}\right)\end{array}$ \\
\hline Fertilized & 6.08 & 86.8 & 5.24 & 7.60 & 5.99 & 49.6 & 9.48 \\
\hline Not fertilized & 5.90 & 84.5 & 5.38 & 7.87 & 5.85 & 48.6 & 8.74 \\
\hline$p$-value ${ }^{3}$ & 0.004 & 0.035 & 0.034 & 0.015 & 0.017 & 0.0003 & $<0.0001$ \\
\hline
\end{tabular}

${ }^{1}$ Means of the fertilized or not fertilized treatment were obtained by averaging data over five tested microgreens grown with four types of hydroponic substrates. ${ }^{2} \mathrm{Mg}=$ magnesium; $\mathrm{S}=$ sulfur; $\mathrm{N}=$ nitrogen; $\mathrm{P}=$ phosphorus. ${ }^{3} p \leq 0.05$ suggests significant difference between means within a column indicated by Tukey's HSD test.

In February 2020, fresh and dry shoot weights were affected by the main effects of species and hydroponic pad type without interaction (Table 4). Ranking for both fresh and dry weights among microgreens are radish $>$ broccoli $>$ kale or cabbage $>$ mustard. Hemp resulted in the highest fresh and dry weights of $1551 \mathrm{~g} \cdot \mathrm{m}^{-2}$ and $101.9 \mathrm{~g} \cdot \mathrm{m}^{-2}$ in microgreens, with jute and biostrate producing similar fresh and dry shoot weights. Micromats resulted in the lowest fresh shoot weight among all substrate types, but similar dry weight to biostrate or jute (Data not shown). Dry weight percentage was not affected by any experimental factor in February.

\subsection{Macronutrient Concentrations}

Macronutrient concentrations including $\mathrm{N}, \mathrm{P}, \mathrm{K}, \mathrm{Ca}, \mathrm{Mg}$, and $\mathrm{S}$ in microgreens were affected by the interaction between species and hydroponic pad in January and February 2020 (Tables 7 and 8). Concentrations of N, P, and K in January and K concentration in February were also affected by the interaction between species and fertilization (Table 6). 
Table 6. Fresh shoot weight, dry weight percentage, and macronutrient concentrations in five microgreens affected by the interaction between species and post-emergent fertilization treatment in January or February 2020.

\begin{tabular}{|c|c|c|c|c|c|c|c|}
\hline \multirow[b]{2}{*}{ Microgreens } & \multirow[b]{2}{*}{ Fertilization } & \multicolumn{5}{|c|}{ January 2020} & \multirow{2}{*}{$\begin{array}{c}\begin{array}{c}\text { February } \\
2020\end{array} \\
\begin{array}{c}K \\
\left(\mathrm{mg} \cdot \mathrm{g}^{-1}\right)\end{array}\end{array}$} \\
\hline & & $\begin{array}{l}\text { Fresh Shoot Weight }{ }^{1,2} \\
\left(\mathrm{~g} \cdot \mathrm{m}^{-2}\right)\end{array}$ & $\begin{array}{l}\text { Dry Weight Percentage } \\
(\%)\end{array}$ & $\begin{array}{c}\mathrm{N}^{3} \\
\left(\mathrm{mg} \cdot \mathrm{g}^{-1}\right)\end{array}$ & $\begin{array}{c}P \\
\left(\mathrm{mg} \cdot \mathrm{g}^{-1}\right)\end{array}$ & $\begin{array}{c}\mathrm{K} \\
\left(\mathrm{mg} \cdot \mathrm{g}^{-1}\right)\end{array}$ & \\
\hline \multirow{2}{*}{ Broccoli } & Fertilized & $1498 \mathrm{c}$ & $6.4 \mathrm{~d}$ & $55.4 \mathrm{~b}$ & $10.1 \mathrm{~cd}$ & $18.8 \mathrm{~b}$ & $14.8 \mathrm{c}$ \\
\hline & Not fertilized & 1408 c & $6.7 \mathrm{~cd}$ & $54.8 \mathrm{~b}$ & $9.9 \mathrm{~d}$ & $16.9 \mathrm{~cd}$ & $13.2 \mathrm{~d}$ \\
\hline \multirow{2}{*}{ Cabbage } & Fertilized & 851 ef & $7.8 \mathrm{a}$ & $51.1 \mathrm{c}$ & $9.5 \mathrm{de}$ & $18.9 \mathrm{~b}$ & $13.3 \mathrm{~d}$ \\
\hline & Not fertilized & $824 \mathrm{f}$ & $8.1 \mathrm{a}$ & $49.2 \mathrm{de}$ & $8.5 \mathrm{f}$ & $17.9 \mathrm{bcd}$ & $11.0 \mathrm{e}$ \\
\hline \multirow{2}{*}{ Kale } & Fertilized & $1112 \mathrm{~d}$ & $6.6 \mathrm{~cd}$ & $50.7 \mathrm{~cd}$ & $9.6 \mathrm{de}$ & $16.2 \mathrm{de}$ & $11.6 \mathrm{e}$ \\
\hline & Not fertilized & $961 \mathrm{e}$ & $7.6 \mathrm{ab}$ & $47.9 \mathrm{e}$ & 8.8 ef & $14.7 \mathrm{ef}$ & $9.5 \mathrm{f}$ \\
\hline \multirow{2}{*}{ Mustard } & Fertilized & $651 \mathrm{~g}$ & $7.9 \mathrm{a}$ & $42.8 \mathrm{f}$ & $12.0 \mathrm{a}$ & $21.6 \mathrm{a}$ & $20.0 \mathrm{a}$ \\
\hline & Not fertilized & $626 \mathrm{~g}$ & $8.0 \mathrm{a}$ & $41.3 \mathrm{~g}$ & $10.8 \mathrm{bc}$ & $18.3 \mathrm{bc}$ & $17.0 \mathrm{~b}$ \\
\hline \multirow{2}{*}{ Radish } & Fertilized & $2235 \mathrm{a}$ & $6.3 \mathrm{~d}$ & $61.0 \mathrm{a}$ & $11.0 \mathrm{~b}$ & $14.5 \mathrm{ef}$ & $10.8 \mathrm{ef}$ \\
\hline & Not fertilized & $2227 \mathrm{~b}$ & $7.1 \mathrm{bc}$ & $60.8 \mathrm{a}$ & $11.2 \mathrm{ab}$ & $14.0 \mathrm{f}$ & $10.3 \mathrm{ef}$ \\
\hline \multirow[t]{3}{*}{$p$-value } & Species & $<0.0001$ & $<0.0001$ & $<0.0001$ & $<0.0001$ & $<0.0001$ & $<0.0001$ \\
\hline & Fertilization & $<0.0001$ & $<0.0001$ & $<0.0001$ & $<0.0001$ & $<0.0001$ & $<0.0001$ \\
\hline & $\begin{array}{l}\text { Species }^{*} \\
\text { fertilization }\end{array}$ & 0.0002 & 0.0012 & 0.0059 & 0.0016 & 0.014 & 0.013 \\
\hline
\end{tabular}

${ }^{1}$ Different lower case letters suggest significant difference among means within a column indicated by the Tukey's HSD test at $p \leq 0.05$.

${ }^{2}$ Each mean was obtained by averaging data over four hydroponic pad types. ${ }^{3} \mathrm{~N}=$ nitrogen; $\mathrm{P}=$ phosphorus; $\mathrm{K}=$ potassium. ${ }^{4}$ Species ${ }^{*}$ fertilization suggests interaction between species and fertilization treatment.

Table 7. Macronutrient concentrations in five microgreens affected by the interaction between species and hydroponic pad type in January 2020.

\begin{tabular}{|c|c|c|c|c|c|c|c|}
\hline \multirow[b]{2}{*}{ Microgreens } & \multirow[b]{2}{*}{ Hydroponic Pad } & \multicolumn{6}{|c|}{ January 2020} \\
\hline & & $\frac{\mathrm{N}}{\left(\mathrm{mg} \cdot \mathrm{g}^{-1}\right)^{1,2,3}}$ & $\begin{array}{c}P \\
\left(\mathrm{mg} \cdot \mathrm{g}^{-1}\right)\end{array}$ & $\begin{array}{c}\mathrm{K} \\
\left(\mathrm{mg} \cdot \mathrm{g}^{-1}\right)\end{array}$ & $\begin{array}{c}\mathrm{Ca} \\
\left(\mathrm{mg} \cdot \mathrm{g}^{-1}\right)\end{array}$ & $\begin{array}{c}\mathrm{Mg} \\
\left(\mathrm{mg} \cdot \mathrm{g}^{-1}\right)\end{array}$ & $\begin{array}{c}\mathrm{S} \\
\left(\mathrm{mg} \cdot \mathrm{g}^{-1}\right)\end{array}$ \\
\hline \multirow{4}{*}{ Broccoli } & Biostrate & $58.1 \mathrm{~b}$ & $10.1 \mathrm{~d}-\mathrm{g}$ & $13.9 \mathrm{de}$ & $7.2 \mathrm{hi}$ & $5.00 \mathrm{~g}-\mathrm{j}$ & 7.07 ef \\
\hline & Hemp & $50.2 \mathrm{fg}$ & $9.7 \mathrm{efg}$ & $30.1 \mathrm{~b}$ & $12.7 \mathrm{~cd}$ & $5.75 \mathrm{c}-\mathrm{f}$ & $9.00 \mathrm{ab}$ \\
\hline & Jute & $55.9 \mathrm{bc}$ & $10.2 \mathrm{~d}-\mathrm{g}$ & $14.0 \mathrm{~d}$ & $11.0 \mathrm{de}$ & $5.53 \mathrm{~d}-\mathrm{h}$ & $8.97 \mathrm{abc}$ \\
\hline & Micro-Mats & $56.2 \mathrm{bc}$ & $9.9 \mathrm{~d}-\mathrm{g}$ & $13.3 \mathrm{~d}-\mathrm{g}$ & $5.4 \mathrm{ij}$ & 4.30 jk & $8.78 \mathrm{a}-\mathrm{d}$ \\
\hline \multirow{4}{*}{ Cabbage } & Biostrate & $57.8 \mathrm{~b}$ & $9.9 \mathrm{~d}-\mathrm{g}$ & $14.4 \mathrm{~d}$ & $9.1 \mathrm{fg}$ & $5.23 \mathrm{f}-\mathrm{i}$ & $8.77 \mathrm{a}-\mathrm{d}$ \\
\hline & Hemp & $41.5 \mathrm{j}$ & $7.8 \mathrm{~h}$ & $35.2 \mathrm{a}$ & $19.6 \mathrm{a}$ & $6.38 \mathrm{bc}$ & $8.11 \mathrm{~b}-\mathrm{e}$ \\
\hline & Jute & $50.2 \mathrm{fg}$ & $8.8 \mathrm{gh}$ & $12.2 \mathrm{~d}-\mathrm{h}$ & $14.4 \mathrm{c}$ & $5.68 c-g$ & $8.68 \mathrm{a}-\mathrm{d}$ \\
\hline & Micro-Mats & $51.1 \mathrm{efg}$ & $9.4 \mathrm{~g}$ & $11.7 \mathrm{~d}-\mathrm{h}$ & $5.1 \mathrm{j}$ & $3.67 \mathrm{k}$ & 9.93 a \\
\hline \multirow{4}{*}{ Kale } & Biostrate & 52.5 def & $9.2 \mathrm{~g}$ & $10.5 \mathrm{gh}$ & $4.9 \mathrm{k}$ & $4.99 \mathrm{~g}-\mathrm{j}$ & $6.02 \mathrm{fg}$ \\
\hline & Hemp & $42.6 \mathrm{ij}$ & $9.3 \mathrm{~g}$ & $31.7 \mathrm{~b}$ & $11.5 \mathrm{de}$ & $6.05 \mathrm{bcd}$ & $8.72 \mathrm{a}-\mathrm{d}$ \\
\hline & Jute & $53.2 \mathrm{de}$ & $9.4 \mathrm{fg}$ & $9.9 \mathrm{~h}$ & $8.9 \mathrm{gh}$ & $5.52 \mathrm{~d}-\mathrm{h}$ & 7.70 cde \\
\hline & Micro-Mats & $48.8 \mathrm{~g}$ & $9.0 \mathrm{gh}$ & $9.8 \mathrm{~h}$ & $3.1 \mathrm{k}$ & $3.78 \mathrm{k}$ & $8.05 b-e$ \\
\hline \multirow{4}{*}{ Mustard } & Biostrate & $45.2 \mathrm{~h}$ & $12.2 \mathrm{ab}$ & $14.4 \mathrm{~d}$ & $11.8 \mathrm{de}$ & $6.55 \mathrm{~b}$ & $7.56 \mathrm{de}$ \\
\hline & Hemp & $35.3 \mathrm{k}$ & $9.3 \mathrm{~g}$ & $37.3 \mathrm{a}$ & $14.4 \mathrm{c}$ & $5.95 \mathrm{~b}-\mathrm{e}$ & $5.89 \mathrm{fg}$ \\
\hline & Jute & 44.7 hi & $12.5 \mathrm{a}$ & $13.7 \mathrm{def}$ & $17.2 \mathrm{~b}$ & $7.59 \mathrm{a}$ & $8.57 \mathrm{bcd}$ \\
\hline & Micro-Mats & $42.8 \mathrm{hij}$ & $11.6 \mathrm{abc}$ & $14.3 \mathrm{~d}$ & $6.4 \mathrm{ij}$ & $4.72 \mathrm{ij}$ & $8.96 \mathrm{abc}$ \\
\hline \multirow{4}{*}{ Radish } & Biostrate & $61.7 \mathrm{a}$ & $11.9 \mathrm{abc}$ & $13.3 \mathrm{~d}-\mathrm{g}$ & $7.2 \mathrm{hi}$ & 5.03 ghi & $4.31 \mathrm{~h}$ \\
\hline & Hemp & $54.7 \mathrm{~cd}$ & $10.8 \mathrm{cde}$ & $21.8 \mathrm{c}$ & $10.8 \mathrm{ef}$ & $5.31 \mathrm{e}-\mathrm{i}$ & $8.66 \mathrm{a}-\mathrm{d}$ \\
\hline & Jute & $63.1 \mathrm{a}$ & $10.7 \mathrm{c}-\mathrm{f}$ & $10.9 \mathrm{fgh}$ & $9.1 \mathrm{fg}$ & 4.84 hij & $5.64 \mathrm{~g}$ \\
\hline & Micro-Mats & $64.1 \mathrm{a}$ & $11.1 \mathrm{bcd}$ & $11.1 \mathrm{e}-\mathrm{h}$ & $5.4 \mathrm{ij}$ & $4.30 \mathrm{jk}$ & $5.34 \mathrm{gh}$ \\
\hline \multirow[t]{3}{*}{$p$-value } & Species & $<0.0001$ & $<0.0001$ & $<0.0001$ & $<0.0001$ & $<0.0001$ & $<0.0001$ \\
\hline & Substrate & $<0.0001$ & $<0.0001$ & $<0.0001$ & $<0.0001$ & $<0.0001$ & $<0.0001$ \\
\hline & $\begin{array}{l}\text { Species* } \\
\text { Substrate }\end{array}$ & $<0.0001$ & $<0.0001$ & $<0.0001$ & $<0.0001$ & $<0.0001$ & $<0.0001$ \\
\hline
\end{tabular}

\footnotetext{
${ }^{1}$ Different lower case letters suggest significant difference among means within a column indicated by the Tukey's HSD test at $p \leq 0.05$.

${ }^{2}$ Each mean was obtained by averaging data over both the fertilized and no-fertilizer treatments. ${ }^{3} \mathrm{~N}=$ nitrogen; $\mathrm{P}=$ phosphorus;

$\mathrm{K}$ = potassium; $\mathrm{Ca}=$ calcium; $\mathrm{Mg}=$ magnesium; $\mathrm{S}=$ sulfur. ${ }^{4}$ Species ${ }^{*}$ Substrate suggests interaction between species and substrate type.
} 
Table 8. Macronutrient concentrations in five microgreens affected by the interaction between species and hydroponic pad in February 2020.

\begin{tabular}{|c|c|c|c|c|c|c|c|}
\hline \multirow[b]{2}{*}{ Microgreens } & \multirow[b]{2}{*}{ Hydroponic Pad } & \multicolumn{6}{|c|}{ February 2020} \\
\hline & & $\frac{\mathrm{N}}{\left(\mathrm{mg} \cdot \mathrm{g}^{-1}\right)^{1,2,3}}$ & $\begin{array}{c}P \\
\left(\mathrm{mg} \cdot \mathrm{g}^{-1}\right)\end{array}$ & $\begin{array}{c}\mathrm{K} \\
\left(\mathrm{mg} \cdot \mathrm{g}^{-1}\right)\end{array}$ & $\begin{array}{c}\mathrm{Ca} \\
\left(\mathrm{mg} \cdot \mathrm{g}^{-1}\right)\end{array}$ & $\begin{array}{c}\mathrm{Mg} \\
\left(\mathrm{mg} \cdot \mathrm{g}^{-1}\right)\end{array}$ & $\begin{array}{c}S \\
\left(\mathrm{mg} \cdot \mathrm{g}^{-1}\right)\end{array}$ \\
\hline \multirow{4}{*}{ Broccoli } & Biostrate & $59.9 \mathrm{ab}$ & 8.7 efg & $13.5 \mathrm{~cd}$ & $8.23 \mathrm{efg}$ & $4.75 \mathrm{e}-\mathrm{h}$ & $6.14 \mathrm{bcd}$ \\
\hline & Hemp & $49.6 \mathrm{fg}$ & $7.6 \mathrm{hi}$ & $19.4 \mathrm{~b}$ & $14.3 \mathrm{ab}$ & $5.20 \mathrm{~b}-\mathrm{e}$ & $7.58 \mathrm{ab}$ \\
\hline & Jute & $57.8 \mathrm{~b}$ & $9.0 \mathrm{~d}-\mathrm{g}$ & $11.6 \mathrm{def}$ & $7.24 \mathrm{f}-\mathrm{i}$ & $4.93 \mathrm{def}$ & $7.0 \mathrm{ab}$ \\
\hline & Micro-Mats & $53.7 \mathrm{de}$ & $8.8 \mathrm{~d}-\mathrm{g}$ & 11.6 def & $3.58 \mathrm{jk}$ & $3.98 \mathrm{ij}$ & $6.78 \mathrm{abc}$ \\
\hline \multirow{4}{*}{ Cabbage } & Biostrate & $50.8 \mathrm{ef}$ & $7.9 \mathrm{gh}$ & $11.8 \mathrm{de}$ & $10.2 \mathrm{de}$ & $4.59 \mathrm{fgh}$ & $2.93 \mathrm{ef}$ \\
\hline & Hemp & $39.3 \mathrm{k}$ & $6.1 \mathrm{j}$ & $19.8 \mathrm{~b}$ & $13.4 \mathrm{bc}$ & $5.07 \mathrm{c}-\mathrm{f}$ & 3.08 ef \\
\hline & Jute & $45.9 \mathrm{hi}$ & $6.7 \mathrm{ij}$ & $8.8 \mathrm{~g}$ & $7.57 \mathrm{e}-\mathrm{h}$ & $4.22 \mathrm{hij}$ & $4.54 \mathrm{def}$ \\
\hline & Micro-Mats & $49.1 \mathrm{fg}$ & $7.5 \mathrm{hi}$ & $8.2 \mathrm{~g}$ & $2.35 \mathrm{k}$ & $2.93 \mathrm{k}$ & $2.72 \mathrm{f}$ \\
\hline \multirow{4}{*}{ Kale } & Biostrate & $47.4 \mathrm{gh}$ & $8.8 \mathrm{def}$ & $9.1 \mathrm{fg}$ & $3.87 \mathrm{jk}$ & $4.68 \mathrm{e}-\mathrm{h}$ & $4.38 \mathrm{def}$ \\
\hline & Hemp & $43.0 \mathrm{ij}$ & $8.5 \mathrm{e}-\mathrm{h}$ & $13.9 \mathrm{~cd}$ & $4.75 \mathrm{ijk}$ & $5.38 \mathrm{bcd}$ & 3.75 ef \\
\hline & Jute & $48.2 \mathrm{fgh}$ & $9.9 \mathrm{~cd}$ & $10.1 \mathrm{efg}$ & 9.49 def & $5.75 \mathrm{~b}$ & $7.93 \mathrm{ab}$ \\
\hline & Micro-Mats & $48.6 \mathrm{fgh}$ & $9.3 c-f$ & $9.1 \mathrm{fg}$ & $3.32 \mathrm{jk}$ & $3.8 \mathrm{ij}$ & $6.94 \mathrm{ab}$ \\
\hline \multirow{4}{*}{ Mustard } & Biostrate & $43.9 \mathrm{ij}$ & $11.9 \mathrm{a}$ & $14.0 \mathrm{~cd}$ & $11.5 \mathrm{~cd}$ & $6.40 \mathrm{a}$ & $6.92 \mathrm{ab}$ \\
\hline & Hemp & 30.31 & $8.3 \mathrm{fgh}$ & $34.6 \mathrm{a}$ & $13.3 \mathrm{bc}$ & $5.52 \mathrm{bc}$ & $6.13 \mathrm{bcd}$ \\
\hline & Jute & $39.7 \mathrm{k}$ & $11.4 \mathrm{ab}$ & 12.4 cde & $16.4 \mathrm{a}$ & $6.75 \mathrm{a}$ & $7.09 \mathrm{ab}$ \\
\hline & Micro-Mats & $42.1 \mathrm{jk}$ & $12.2 \mathrm{a}$ & $13.1 \mathrm{~cd}$ & $5.75 \mathrm{~g}-\mathrm{j}$ & 4.33 ghi & $8.55 \mathrm{a}$ \\
\hline \multirow{4}{*}{ Radish } & Biostrate & $61.3 \mathrm{a}$ & $9.9 \mathrm{~cd}$ & $10.2 \mathrm{efg}$ & 5.52 hij & $4.58 \mathrm{fgh}$ & $4.48 \mathrm{def}$ \\
\hline & Hemp & $54.7 \mathrm{~cd}$ & $9.9 \mathrm{~cd}$ & $14.6 \mathrm{c}$ & $10.1 \mathrm{de}$ & $4.90 \mathrm{~d}-\mathrm{g}$ & $6.87 \mathrm{ab}$ \\
\hline & Jute & $58.4 \mathrm{ab}$ & $10.3 \mathrm{bc}$ & $8.5 \mathrm{~g}$ & 8.53 ef & $4.76 \mathrm{e}-\mathrm{h}$ & 4.86 cde \\
\hline & Micro-Mats & $57.4 \mathrm{bc}$ & 9.5 cde & $9.0 \mathrm{~g}$ & $4.49 \mathrm{jk}$ & $3.74 \mathrm{j}$ & $4.53 \mathrm{def}$ \\
\hline \multirow[t]{3}{*}{$p$-value } & Species & $<0.0001$ & $<0.0001$ & $<0.0001$ & $<0.0001$ & $<0.0001$ & $<0.0001$ \\
\hline & Substrate & $<0.0001$ & $<0.0001$ & $<0.0001$ & $<0.0001$ & $<0.0001$ & $<0.0001$ \\
\hline & $\begin{array}{c}\text { Species }^{*} \\
\text { Substrate }\end{array}$ & $<0.0001$ & $<0.0001$ & $<0.0001$ & $<0.0001$ & $<0.0001$ & $<0.0001$ \\
\hline
\end{tabular}

${ }^{1}$ Different lower case letters suggest significant difference among means within a column indicated by the Tukey's HSD test at $p \leq 0.05$.

${ }^{2}$ Each mean was obtained by averaging data over both the fertilized and no-fertilizer treatments. ${ }^{3} \mathrm{~N}=$ nitrogen; $\mathrm{P}=$ phosphorus;

$\mathrm{K}=$ potassium; $\mathrm{Ca}=$ calcium; $\mathrm{Mg}=$ magnesium; $\mathrm{S}=$ sulfur. ${ }^{4}$ Species * Substrate suggests interaction between species and substrate type.

\subsubsection{Nitrogen}

Nitrogen concentration ranged from $35.3 \mathrm{mg} \cdot \mathrm{g}^{-1}$ in mustard grown with hemp to $64.1 \mathrm{mg} \cdot \mathrm{g}^{-1}$ in radish grown with micro-mats in January 2020 (Table 7) and from $30.3 \mathrm{mg} \cdot \mathrm{g}^{-1}$ in mustard grown with hemp to $61.3 \mathrm{mg} \cdot \mathrm{g}^{-1}$ in radish grown with biostrate in February 2020 (Table 8).

Radish microgreens had higher $\mathrm{N}$ concentration than other microgreen species when grown with any substrate type in January 2020 ranging from $54.7 \mathrm{mg} \cdot \mathrm{g}^{-1}$ in hemp to $64.1 \mathrm{mg} \cdot \mathrm{g}^{-1}$ in micro-mats. Mustard had the lowest $\mathrm{N}$ concentration among all microgreens grown with each substrate in both experiments ranging from $35.3 \mathrm{mg} \cdot \mathrm{g}^{-1}$ in hemp to $45.2 \mathrm{mg} \cdot \mathrm{g}^{-1}$ in biostrate in January, and from $30.3 \mathrm{mg} \cdot \mathrm{g}^{-1}$ in hemp mat to $43.9 \mathrm{mg} \cdot \mathrm{g}^{-1}$ in biostrate in February. In February, higher $\mathrm{N}$ concentrations were found in radish and broccoli than kale, cabbage, or mustard when grown with any substrate type. Hemp resulted in lower $\mathrm{N}$ concentrations in all five microgreens than the other three substrates in both experiments. One time post-emergent fertilization increased overall $\mathrm{N}$ concentration by $2.0 \%$ in February 2020 (Table 5).

\subsubsection{Phosphorus}

Phosphorus concentration ranged from $7.8 \mathrm{mg} \cdot \mathrm{g}^{-1}$ in cabbage grown with hemp to $12.5 \mathrm{mg} \cdot \mathrm{g}^{-1}$ in mustard grown with a jute mat in January 2020 (Table 7 ) and from 
$6.1 \mathrm{mg} \cdot \mathrm{g}^{-1}$ in cabbage grown with hemp to $12.2 \mathrm{mg} \cdot \mathrm{g}^{-1}$ in mustard grown with micromats in February 2020 (Table 8).

In January, four substrate types generally resulted in similar P concentrations in each microgreen, except that $\mathrm{P}$ concentration in mustard was lower when grown with hemp than other substrate types. Ranking of $P$ concentration among microgreens varied in different substrate types, where mustard had the highest $\mathrm{P}$ concentrations of $12.2 \mathrm{mg} \cdot \mathrm{g}^{-1}$, $12.5 \mathrm{mg} \cdot \mathrm{g}^{-1}$, and $11.6 \mathrm{mg} \cdot \mathrm{g}^{-1}$ among the five species in three substrates including biostrate, jute mat, and micro-mats, respectively (Table 7). Phosphorus concentration in January was also affected by the interaction between microgreen species and fertilization treatment, where fertilization increased $\mathrm{P}$ concentration in cabbage and mustard, but resulted in similar P concentrations in broccoli, kale, and radish (Table 6).

In February, mustard grown with biostrate, jute, and micro-mats had comparable highest $\mathrm{P}$ concentrations ranging from 11.4 to $12.2 \mathrm{mg} \cdot \mathrm{g}^{-1}$ (Table 8 ). Hemp resulted in the lowest $\mathrm{P}$ concentrations of $7.6 \mathrm{mg} \cdot \mathrm{g}^{-1}$ and $8.3 \mathrm{mg} \cdot \mathrm{g}^{-1}$ compared to other substrate in broccoli and mustard, respectively. Fertilization increased overall $\mathrm{P}$ concentration by $8.4 \%$ compared to the no-fertilization treatment (Table 5).

\subsubsection{Potassium}

Potassium concentration ranged from $9.8 \mathrm{mg} \cdot \mathrm{g}^{-1}$ in kale grown with micro-mats to $37.3 \mathrm{mg} \cdot \mathrm{g}^{-1}$ in mustard grown with a hemp mat in January 2020 (Table 7) and from $8.2 \mathrm{mg} \cdot \mathrm{g}^{-1}$ in cabbage grown with micro-mats to $34.6 \mathrm{mg} \cdot \mathrm{g}^{-1}$ in mustard grown with a hemp mat in February 2020 (Table 8).

Hemp resulted in higher $\mathrm{K}$ concentrations than other substrate types in each microgreen in both experiments. Among all treatment combinations, the highest $\mathrm{K}$ concentrations were found in mustard grown with hemp in both experiments, $37.3 \mathrm{mg} \cdot \mathrm{g}^{-1}$ in January and $34.6 \mathrm{mg} \cdot \mathrm{g}^{-1}$ in February respectively. Cabbage had comparable K concentration with mustard in each substrate type in January. In February, broccoli and cabbage grown with hemp had the second highest $\mathrm{K}$ concentrations next to mustard grown with hemp.

Potassium concentration was also affected by the interaction between species and fertilization in January, where fertilization increased $\mathrm{K}$ concentrations in broccoli and mustard by $11.2 \%$ and $18.0 \%$, but not in the other three microgreens (Table 6 ).

\subsubsection{Calcium}

Calcium concentration ranged from $3.12 \mathrm{mg} \cdot \mathrm{g}^{-1}$ in kale grown with micro-mats to $19.6 \mathrm{mg} \cdot \mathrm{g}^{-1}$ in cabbage grown with a hemp mat in January 2020 (Table 7) and from $2.35 \mathrm{mg} \cdot \mathrm{g}^{-1}$ in cabbage grown with micro-mats to $16.4 \mathrm{mg} \cdot \mathrm{g}^{-1}$ in mustard grown with a jute mat in February 2020 (Table 8).

The five microgreens varied in their Ca concentrations, and substrate type altered concentrations for each species. In January, cabbage and mustard had higher Ca concentrations than broccoli, kale, or radish when grown with biostrate, hemp, or jute mat. When grown with micro-mats, broccoli had higher Ca concentrations than the other four microgreens. The hemp mat resulted in the highest Ca concentrations of $19.6 \mathrm{mg} \cdot \mathrm{g}^{-1}$ in cabbage and $11.5 \mathrm{mg} \cdot \mathrm{g}^{-1}$ in kale, whereas the jute mat resulted in the highest Ca concentration of $17.2 \mathrm{mg} \cdot \mathrm{g}^{-1}$ in mustard.

There was not as much separation of means in February, where broccoli, cabbage, kale, and radish had similar Ca concentrations when grown with a jute mat or micromats. The hemp mat resulted in higher Ca concentrations in broccoli $\left(14.3 \mathrm{mg} \cdot \mathrm{g}^{-1}\right)$ and cabbage $\left(13.4 \mathrm{mg} \cdot \mathrm{g}^{-1}\right)$ than other substrates, whereas the jute mat resulted in higher $\mathrm{Ca}$ concentrations in kale $\left(9.49 \mathrm{mg} \cdot \mathrm{g}^{-1}\right)$ and mustard $\left(16.4 \mathrm{mg} \cdot \mathrm{g}^{-1}\right)$ than other substrate types.

\subsubsection{Magnesium}

Magnesium concentration ranged from $3.67 \mathrm{mg} \cdot \mathrm{g}^{-1}$ in cabbage grown with micromats to $7.59 \mathrm{mg} \cdot \mathrm{g}^{-1}$ in mustard grown with a jute mat in January 2020 (Table 7) and from 
$2.93 \mathrm{mg} \cdot \mathrm{g}^{-1}$ in cabbage grown in micro-mats to $6.75 \mathrm{mg} \cdot \mathrm{g}^{-1}$ in mustard grown with a jute mate in February 2020 (Table 8).

Broccoli, cabbage, and kale had similar Mg concentrations when grown with any of the four substrate types in January, and grown with biostrate or a hemp mat in February. Mustard had higher Mg concentrations than other microgreens when grown with biostrate or a jute mat in both experiments. Micro-mats resulted in lower $\mathrm{Mg}$ concentrations than the other three substrate types in each microgreen in January, and in broccoli, kale, cabbage, and mustard in February. One time fertilization decreased $\mathrm{Mg}$ concentration by $2.6 \%$ compared with the no-fertilization treatment (Table 5).

\subsubsection{Sulfur}

Sulfur concentration ranged from $4.31 \mathrm{mg} \cdot \mathrm{g}^{-1}$ in radish grown with biostrate to $9.93 \mathrm{mg} \cdot \mathrm{g}^{-1}$ in cabbage grown with micro-mats in January 2020 (Table 7) and from $2.72 \mathrm{mg} \cdot \mathrm{g}^{-1}$ in cabbage grown with micro-mats to $8.55 \mathrm{mg} \cdot \mathrm{g}^{-1}$ in mustard grown with micro-mats in February 2020 (Table 8), affected by the interaction between species and substrate interaction in both experiments.

In general, separation among microgreens within a substrate type, or among substrates within one microgreen were not as much in January or February. In January, radish had lower $\mathrm{S}$ concentrations of $4.31 \mathrm{mg} \cdot \mathrm{g}^{-1}$ to $5.64 \mathrm{mg} \cdot \mathrm{g}^{-1}$ than other microgreens when grown with biostrate, a jute mat, or micro-mats. The hemp mat, jute mat, and micro-mats resulted in similar $\mathrm{S}$ concentrations in broccoli (8.78-9.00 $\left.\mathrm{mg} \cdot \mathrm{g}^{-1}\right)$ and kale $\left(7.70-8.72 \mathrm{mg} \cdot \mathrm{g}^{-1}\right)$. One time fertilization decreased $\mathrm{S}$ concentration by $3.6 \%$ compared with the no-fertilization treatment (Table 5). In February, four substrate types resulted in similar S concentrations in broccoli (6.14-7.58 $\left.\mathrm{mg} \cdot \mathrm{g}^{-1}\right)$ and cabbage $\left(2.72-4.54 \mathrm{mg} \cdot \mathrm{g}^{-1}\right)$. Kale and cabbage had lower $\mathrm{S}$ concentrations than other microgreens when grown in a hemp mat. Broccoli, kale, and mustard had similar $\mathrm{S}$ concentrations when grown with a jute mat or micro-mats (Table 8).

\subsection{Micronutrient Concentrations}

Micronutrient concentrations including $\mathrm{Cu}, \mathrm{Fe}, \mathrm{Mn}, \mathrm{Zn}$, and $\mathrm{B}$ were all affected by the interaction between species and hydroponic pad type, but not affected by the fertilization treatment.

\subsubsection{Copper}

Copper concentration ranged from 0.51 to $1.92 \mu \mathrm{g} \cdot \mathrm{g}^{-1}$ in mustard grown with a hemp mat in January 2020 (Table 9) and from 0.39 to $5.09 \mu \mathrm{g} \cdot \mathrm{g}^{-1}$ in cabbage grown in a hemp mat in February 2020 (Table 10).

Table 9. Micronutrient concentrations in five microgreens affected by the interaction between species and hydroponic pad type in January 2020.

\begin{tabular}{|c|c|c|c|c|c|c|}
\hline \multirow[b]{2}{*}{ Microgreens } & \multirow[b]{2}{*}{ Hydroponic Pad } & \multicolumn{5}{|c|}{ January 2020} \\
\hline & & $\frac{\mathrm{Cu}}{\left(\mu \mathrm{g} \cdot \mathrm{g}^{-1}\right)^{1,2,3}}$ & $\begin{array}{c}\text { Fe } \\
\left(\mu g \cdot g^{-1}\right)\end{array}$ & $\begin{array}{c}\text { Mn } \\
\left(\mu g \cdot g^{-1}\right)\end{array}$ & $\begin{array}{c}\mathrm{Zn} \\
\left(\mu \mathrm{g} \cdot \mathrm{g}^{-1}\right)\end{array}$ & $\begin{array}{c}\text { B } \\
\left(\mu \mathrm{g} \cdot \mathrm{g}^{-1}\right)\end{array}$ \\
\hline \multirow{4}{*}{ Broccoli } & Biostrate & $0.51 \mathrm{~d}$ & $94.8 \mathrm{~d}-\mathrm{i}$ & $35.1 \mathrm{i}-1$ & $78.8 \mathrm{de}$ & $16.5 \mathrm{~h}-\mathrm{k}$ \\
\hline & Hemp & $0.51 \mathrm{~d}$ & $86.2 \mathrm{f}-\mathrm{i}$ & 45.7 ghi & $74.4 \mathrm{def}$ & $34.6 \mathrm{bc}$ \\
\hline & Jute & $0.71 \mathrm{~cd}$ & 119.5 bc & $62.7 \mathrm{de}$ & $110.5 \mathrm{~b}$ & $23.9 \mathrm{e}-\mathrm{h}$ \\
\hline & Micro-Mats & $0.51 \mathrm{~d}$ & $116.9 \mathrm{bcd}$ & $33.4 \mathrm{jkl}$ & $87.3 \mathrm{~cd}$ & $17.1 \mathrm{~g}-\mathrm{j}$ \\
\hline \multirow{4}{*}{ Cabbage } & Biostrate & $0.51 \mathrm{~d}$ & $113.3 \mathrm{~b}-\mathrm{e}$ & 58.4 def & $76.1 \mathrm{de}$ & $19.9 \mathrm{f}-\mathrm{i}$ \\
\hline & Hemp & $0.51 \mathrm{~d}$ & $134.7 \mathrm{ab}$ & $118.6 \mathrm{a}$ & $84.8 \mathrm{~d}$ & $45.0 \mathrm{a}$ \\
\hline & Jute & $0.51 \mathrm{~d}$ & $156.3 \mathrm{a}$ & $116.5 \mathrm{a}$ & $133.3 \mathrm{a}$ & 28.0 cde \\
\hline & Micro-Mats & $0.51 \mathrm{~d}$ & $134 \mathrm{ab}$ & 51.5 efg & 78.5 de & $23.9 \mathrm{~d}-\mathrm{g}$ \\
\hline \multirow{4}{*}{ Kale } & Biostrate & $1.29 \mathrm{bc}$ & $84.8 \mathrm{c}-\mathrm{h}$ & 45.3 ghi & 53.3 hij & $9.65 \mathrm{k}$ \\
\hline & Hemp & $0.67 \mathrm{~d}$ & $97.4 \mathrm{c}-\mathrm{h}$ & $65.9 \mathrm{~cd}$ & $68.6 \mathrm{efg}$ & $37.3 \mathrm{~b}$ \\
\hline & Jute & $1.29 \mathrm{bc}$ & $90.3 \mathrm{e}-\mathrm{i}$ & $74.3 \mathrm{c}$ & 75.3 def & $14.5 \mathrm{ijk}$ \\
\hline & Micro-Mats & $1.29 \mathrm{bc}$ & $90.4 \mathrm{e}-\mathrm{i}$ & $39.0 \mathrm{~h}-\mathrm{k}$ & $50.8 \mathrm{ij}$ & $11.9 \mathrm{jk}$ \\
\hline
\end{tabular}


Table 9. Cont

\begin{tabular}{|c|c|c|c|c|c|c|}
\hline \multirow[b]{2}{*}{ Microgreens } & \multirow[b]{2}{*}{ Hydroponic Pad } & \multicolumn{5}{|c|}{ January 2020} \\
\hline & & $\begin{array}{c}\mathrm{Cu} \\
\left(\mu \mathrm{g} \cdot \mathrm{g}^{-1}\right)^{1,2,3}\end{array}$ & $\begin{array}{c}\text { Fe } \\
\left(\mu \mathrm{g} \cdot \mathrm{g}^{-1}\right)\end{array}$ & $\begin{array}{c}\mathrm{Mn} \\
\left(\mu \mathrm{g} \cdot \mathrm{g}^{-1}\right)\end{array}$ & $\begin{array}{c}\mathrm{Zn} \\
\left(\mu \mathrm{g} \cdot \mathrm{g}^{-1}\right)\end{array}$ & $\begin{array}{c}\text { B } \\
\left(\mu g \cdot g^{-1}\right)\end{array}$ \\
\hline \multirow{4}{*}{ Mustard } & Biostrate & $1.29 \mathrm{bc}$ & $107.3 \mathrm{c}-\mathrm{f}$ & $49.4 \mathrm{fgh}$ & $67.6 \mathrm{e}-\mathrm{h}$ & 26.3 def \\
\hline & Hemp & $1.92 \mathrm{a}$ & $101 \mathrm{c}-\mathrm{g}$ & $42.7 \mathrm{~g}-\mathrm{k}$ & $61.1 \mathrm{f}-\mathrm{i}$ & $52.0 \mathrm{a}$ \\
\hline & Jute & $1.29 \mathrm{bc}$ & $112.8 \mathrm{~b}-\mathrm{e}$ & $88.4 \mathrm{~b}$ & $100.5 \mathrm{bc}$ & $30.4 \mathrm{bcd}$ \\
\hline & Micro-Mats & $1.29 \mathrm{bc}$ & $107 \mathrm{c}-\mathrm{f}$ & $33.4 \mathrm{jkl}$ & $59.6 \mathrm{~g}-\mathrm{j}$ & 24.4 def \\
\hline \multirow{4}{*}{ Radish } & Biostrate & $1.72 \mathrm{ab}$ & 83.4 ghi & $31.5 \mathrm{kl}$ & $53.6 \mathrm{hij}$ & $11.5 \mathrm{jk}$ \\
\hline & Hemp & $1.29 \mathrm{bc}$ & $79.8 \mathrm{ghi}$ & $44.6 \mathrm{~g}-\mathrm{j}$ & $57.4 \mathrm{~g}-\mathrm{j}$ & $22.8 \mathrm{f}-\mathrm{h}$ \\
\hline & Jute & $1.29 \mathrm{bc}$ & $76.9 \mathrm{hi}$ & $44.3 \mathrm{~g}-\mathrm{j}$ & $65.3 \mathrm{e}-\mathrm{i}$ & $10.6 \mathrm{jk}$ \\
\hline & Micro-Mats & $1.29 \mathrm{bc}$ & $74.0 \mathrm{i}$ & 25.31 & $47.3 \mathrm{j}$ & $11.5 \mathrm{jk}$ \\
\hline \multirow[t]{3}{*}{$p$-value } & Species & $<0.0001$ & $<0.0001$ & $<0.0001$ & $<0.0001$ & $<0.0001$ \\
\hline & Substrate & 0.5972 & $<0.0001$ & $<0.0001$ & $<0.0001$ & $<0.0001$ \\
\hline & Species $*$ Substrate ${ }^{4}$ & $<0.0001$ & $<0.0001$ & $<0.0001$ & $<0.0001$ & $<0.0001$ \\
\hline
\end{tabular}

${ }^{1}$ Different lower case letters suggest significant difference among means within a column indicated by the Tukey's HSD test at $p \leq 0.05$.

2 Each mean was obtained by averaging data over both the fertilized and no-fertilizer treatments. ${ }^{3} \mathrm{Cu}=\mathrm{copper} ; \mathrm{Fe}=\mathrm{iron} ; \mathrm{Mn}=\mathrm{manganese}$;

$\mathrm{Zn}=$ zinc; $\mathrm{B}=$ boron. ${ }^{4}$ Species * Substrate suggests interaction between species and substrate type.

Table 10. Micronutrient concentrations in five microgreens affected by the interaction between species and hydroponic pad type in February 2020.

\begin{tabular}{|c|c|c|c|c|c|c|}
\hline \multirow[b]{2}{*}{ Microgreens } & \multirow[b]{2}{*}{ Hydroponic Pad } & \multicolumn{5}{|c|}{ February 2020} \\
\hline & & $\frac{\mathrm{Cu}}{\left(\mu \mathrm{g} \cdot \mathrm{g}^{-1}\right)^{1,2,3}}$ & $\begin{array}{c}\text { Fe } \\
\left(\mu g \cdot g^{-1}\right)\end{array}$ & $\begin{array}{c}\text { Mn } \\
\left(\mu g \cdot g^{-1}\right)\end{array}$ & $\begin{array}{c}\mathrm{Zn} \\
\left(\mu \mathrm{g} \cdot \mathrm{g}^{-1}\right)\end{array}$ & $\begin{array}{c}\text { B } \\
\left(\mu g \cdot g^{-1}\right)\end{array}$ \\
\hline \multirow{4}{*}{ Broccoli } & Biostrate & $2.82 \mathrm{abc}$ & $80.4 \mathrm{~b}-\mathrm{e}$ & $29.2 \mathrm{f}-\mathrm{i}$ & $63.7 \mathrm{bc}$ & $12.9 \mathrm{f}-\mathrm{k}$ \\
\hline & Hemp & $2.82 \mathrm{abc}$ & $71.1 \mathrm{def}$ & $40.8 \mathrm{c}-\mathrm{f}$ & $60.0 \mathrm{bcd}$ & $26.4 \mathrm{bc}$ \\
\hline & Jute & $1.20 \mathrm{bcd}$ & $81.2 \mathrm{~b}-\mathrm{e}$ & $47.5 \mathrm{bc}$ & $68.0 \mathrm{ab}$ & $19.5 \mathrm{c}-\mathrm{g}$ \\
\hline & Micro-Mats & $0.82 \mathrm{~cd}$ & $75.9 \mathrm{c}-\mathrm{f}$ & $25.8 \mathrm{hi}$ & $55.6 \mathrm{bcd}$ & $16.5 \mathrm{e}-\mathrm{j}$ \\
\hline \multirow{4}{*}{ Cabbage } & Biostrate & $3.50 \mathrm{ab}$ & $62.7 \mathrm{fg}$ & $34.9 \mathrm{e}-\mathrm{h}$ & $24.8 \mathrm{hg}$ & $12.0 \mathrm{~g}-\mathrm{k}$ \\
\hline & Hemp & $5.09 \mathrm{a}$ & 70.6 def & $56.6 \mathrm{~b}$ & $30.9 \mathrm{fgh}$ & $21.3 \mathrm{~b}-\mathrm{f}$ \\
\hline & Jute & $4.92 \mathrm{a}$ & 99.9 a & $71.8 \mathrm{a}$ & $61.3 \mathrm{bcd}$ & $18.3 \mathrm{c}-\mathrm{h}$ \\
\hline & Micro-Mats & $0.88 \mathrm{~cd}$ & $41.4 \mathrm{~h}$ & 27.5 ghi & $17.0 \mathrm{~h}$ & $7.7 \mathrm{k}$ \\
\hline \multirow{4}{*}{ Kale } & Biostrate & $0.50 \mathrm{~cd}$ & $42.6 \mathrm{~h}$ & $37.5 c-h$ & $26.3 \mathrm{hg}$ & 9.7 ijk \\
\hline & Hemp & $4.26 \mathrm{a}$ & $42.8 \mathrm{~h}$ & $48.2 \mathrm{bc}$ & $21.0 \mathrm{hg}$ & $15.2 \mathrm{e}-\mathrm{j}$ \\
\hline & Jute & $0.39 \mathrm{~d}$ & $83.3 \mathrm{bcd}$ & $76.3 \mathrm{a}$ & $70.2 \mathrm{ab}$ & $17.8 \mathrm{~d}-\mathrm{i}$ \\
\hline & Micro-Mats & $0.39 \mathrm{~d}$ & 65.7 efg & $37.8 \mathrm{c}-\mathrm{g}$ & 45.4 def & $11.0 \mathrm{~h}-\mathrm{k}$ \\
\hline \multirow{4}{*}{ Mustard } & Biostrate & $0.39 \mathrm{~d}$ & $94.8 \mathrm{ab}$ & 43.5 cde & $66.4 \mathrm{ab}$ & $25.7 \mathrm{bcd}$ \\
\hline & Hemp & $0.39 \mathrm{~d}$ & $69.0 \mathrm{~d}-\mathrm{g}$ & $32.9 \mathrm{e}-\mathrm{h}$ & 48.7 cde & $37.9 \mathrm{a}$ \\
\hline & Jute & $0.71 \mathrm{~cd}$ & $90.1 \mathrm{abc}$ & 81.9 a & $83.3 \mathrm{a}$ & $28.2 \mathrm{~b}$ \\
\hline & Micro-Mats & $0.39 \mathrm{~d}$ & $94.5 \mathrm{ab}$ & $29.1 \mathrm{f}-\mathrm{i}$ & $55.4 \mathrm{bcd}$ & $23.4 \mathrm{~b}-\mathrm{e}$ \\
\hline \multirow{4}{*}{ Radish } & Biostrate & $0.39 \mathrm{~d}$ & $54.3 \mathrm{gh}$ & $20.2 \mathrm{i}$ & 48.6 cde & $15.4 \mathrm{e}-\mathrm{k}$ \\
\hline & Hemp & $0.39 \mathrm{~d}$ & $44.5 \mathrm{~h}$ & $45.5 \mathrm{bcd}$ & $47.1 \mathrm{c}-\mathrm{f}$ & $15.0 \mathrm{e}-\mathrm{k}$ \\
\hline & Jute & $0.39 \mathrm{~d}$ & $53.7 \mathrm{gh}$ & 42.4 cde & $61.9 \mathrm{bcd}$ & $10.2 \mathrm{~h}-\mathrm{k}$ \\
\hline & Micro-Mats & $0.39 \mathrm{~d}$ & $40.6 \mathrm{~h}$ & $19.4 \mathrm{i}$ & $37.1 \mathrm{efg}$ & $8.7 \mathrm{jk}$ \\
\hline \multirow[t]{3}{*}{$p$-value } & Species & $<0.0001$ & $<0.0001$ & $<0.0001$ & $<0.0001$ & $<0.0001$ \\
\hline & Substrate & $<0.0001$ & $<0.0001$ & $<0.0001$ & $<0.0001$ & $<0.0001$ \\
\hline & Species $*$ Substrate ${ }^{4}$ & $<0.0001$ & $<0.0001$ & $<0.0001$ & $<0.0001$ & $<0.0001$ \\
\hline
\end{tabular}

${ }^{1}$ Different lower case letters suggest significant difference among means within a column indicated by the Tukey's HSD test at $p \leq 0.05$.

${ }^{2}$ Each mean was obtained by averaging data over both the fertilized and no-fertilizer treatments. ${ }^{3} \mathrm{Cu}=$ copper; $\mathrm{Fe}=$ iron; $\mathrm{Mn}=$ manganese;

$\mathrm{Zn}=$ zinc; $\mathrm{B}=$ boron. ${ }^{4}$ Species * Substrate suggests interaction between species and substrate type.

Four hydroponic pad types resulted in similar $\mathrm{Cu}$ concentrations in radish and broccoli in both experiments, in cabbage in January, in mustard in February. Biostrate, jute mate, 
and micro-mats resulted in similar $\mathrm{Cu}$ concentrations in Kale in both experiments and in mustard in January.

Radish, kale, and mustard had similar $\mathrm{Cu}$ concentrations when grown with biostrate or micro-mats, higher than broccoli or cabbage in January but lower than broccoli or cabbage in February when grown with biostrate. When grown with a hemp mat, broccoli, kale, and cabbage had similar $\mathrm{Cu}$ concentrations in both experiments. Micro-mats resulted in similar $\mathrm{Cu}$ concentrations among all five microgreens in February.

\subsubsection{Iron}

Iron concentration ranged from $74.0 \mu \mathrm{g} \cdot \mathrm{g}^{-1}$ in radish grown with micro-mats to $156.3 \mu \mathrm{g} \cdot \mathrm{g}^{-1}$ in cabbage grown with a jute mat in January 2020 (Table 9) and from $40.6 \mu \mathrm{g} \cdot \mathrm{g}^{-1}$ in radish grown with micro-mats to $99.9 \mu \mathrm{g} \cdot \mathrm{g}^{-1}$ in cabbage grown with a jute mat in February 2020 (Table 10).

Four substrates resulted in similar Fe concentrations in radish, kale, and mustard in January and in radish and broccoli in February. Cabbage grown with a jute mat had the highest Fe concentrations of $156.3 \mu \mathrm{g} \cdot \mathrm{g}^{-1}$ in January and $99.9 \mu \mathrm{g} \cdot \mathrm{g}^{-1}$ in February among all treatment combinations, respectively. In addition, cabbage grown with a hemp mat and micro-mats also had higher Fe concentrations than other microgreens in January 2020. Mustard had higher Fe concentrations of $94.8 \mu \mathrm{g} \cdot \mathrm{g}^{-1}$ and $94.5 \mu \mathrm{g} \cdot \mathrm{g}^{-1}$ than other microgreens when grown with biostrate and micro-mats in February.

\subsubsection{Manganese}

Manganese concentration ranged from $25.3 \mu \mathrm{g} \cdot \mathrm{g}^{-1}$ in radish grown with micro-mats to $118.6 \mu \mathrm{g} \cdot \mathrm{g}^{-1}$ in cabbage grown with a hemp mat in January 2020 (Table 9) and from $19.4 \mu \mathrm{g} \cdot \mathrm{g}^{-1}$ in radish grown with micro-mats to $81.9 \mu \mathrm{g} \cdot \mathrm{g}^{-1}$ in mustard grown with a jute mat in February 2020 (Table 10).

In January, cabbage had higher Mn concentrations of $118.6 \mu \mathrm{g} \cdot \mathrm{g}^{-1}, 116.5 \mu \mathrm{g} \cdot \mathrm{g}^{-1}$, and $51.5 \mu \mathrm{g} \cdot \mathrm{g}^{-1}$ than other microgreens when grown with hemp, jute, or micro-mats, respectively. Hemp and jute mats resulted in similar Mn concentrations in kale, cabbage, and radish, higher than those in biostrate or micro-mats.

In February, hemp and jute mats resulted in higher Mn concentrations than biostrate or micro-mats in kale, cabbage, and radish, while a jute mat resulted in the highest $\mathrm{Mn}$ concentrations in broccoli and mustard.

\subsubsection{Zinc}

Zinc concentration ranged from $47.3 \mu \mathrm{g} \cdot \mathrm{g}^{-1}$ in radish grown with micro-mats to $133.3 \mu \mathrm{g} \cdot \mathrm{g}^{-1}$ in cabbage grown with a jute mat in January 2020 (Table 9) and from $17.0 \mu \mathrm{g} \cdot \mathrm{g}^{-1}$ in cabbage grown with micro-mats to $83.3 \mu \mathrm{g} \cdot \mathrm{g}^{-1}$ in mustard grown with a jute mat in February 2020 (Table 10).

In January, broccoli and cabbage had higher Zn concentrations than kale, mustard, or radish when grown with a jute mat or micro-mats. The jute mat resulted in the highest $\mathrm{Zn}$ concentration of $110.5 \mu \mathrm{g} \cdot \mathrm{g}^{-1}, 133.3 \mu \mathrm{g} \cdot \mathrm{g}^{-1}$, and $100.5 \mu \mathrm{g} \cdot \mathrm{g}^{-1}$ than the other three substrate types in broccoli, cabbage, and mustard, respectively.

In February, jute mat also resulted in higher Zn concentrations of $61.3 \mu \mathrm{g} \cdot \mathrm{g}^{-1}, 70.2 \mu \mathrm{g} \cdot \mathrm{g}^{-1}$, and $83.3 \mu \mathrm{g} \cdot \mathrm{g}^{-1}$ than other substrate types in kale, cabbage, and mustard, respectively. Separation among microgreens was not as much when grown with a certain substrate.

\subsubsection{Boron}

Boron concentration ranged from $9.65 \mu \mathrm{g} \cdot \mathrm{g}^{-1}$ in kale grown with biostrate to $45.0 \mu \mathrm{g} \cdot \mathrm{g}^{-1}$ in cabbage grown with a hemp mat in January 2020 (Table 9) and from $7.7 \mu \mathrm{g} \cdot \mathrm{g}^{-1}$ in cabbage grown with micro-mats to $37.9 \mu \mathrm{g} \cdot \mathrm{g}^{-1}$ in mustard grown with a hemp mat in February 2020 (Table 10).

In January, cabbage had higher B concentrations of $45.0 \mu \mathrm{g} \cdot \mathrm{g}^{-1}$ and $28.0 \mu \mathrm{g} \cdot \mathrm{g}^{-1}$ than other microgreens when grown with a hemp or jute mat, respectively. Kale, mustard, and 
radish grown with biostrate, jute, and micro-mats had similar B concentrations, lower than most other treatment combinations. Hemp mat resulted in higher B concentrations of $34.6 \mu \mathrm{g} \cdot \mathrm{g}^{-1}, 45.0 \mu \mathrm{g} \cdot \mathrm{g}^{-1}, 37.3 \mu \mathrm{g} \cdot \mathrm{g}^{-1}, 52.0 \mu \mathrm{g} \cdot \mathrm{g}^{-1}$, and $22.8 \mu \mathrm{g} \cdot \mathrm{g}^{-1}$ than other substrates in broccoli, cabbage, kale, mustard, and radish, respectively.

In February, mustard had higher B concentrations of $23.4 \mu \mathrm{g} \cdot \mathrm{g}^{-1}$ in micro-mats to $37.9 \mu \mathrm{g} \cdot \mathrm{g}^{-1}$ in jute mat than other microgreens when grown with each hydroponic pad type. Hemp resulted in higher B concentrations than other substrates in broccoli and mustard.

\section{Discussion}

Fresh shoot yield is of the most limiting factor in microgreen production [4,20,23-25]. Microgreens varied in their fresh shoot yield, which was altered by hydroponic substrate type and fertilization treatment. Fresh, dry shoot weights, and dry weight percentage of tested microgreens in this current study are in similar ranges as our previous study when similar microgreen species/varieties were grown with a peat-based soilless substrate [26], suggesting selection of high-yielding microgreen species or varieties can potentially be applied to different growing systems including using peat-based substrate, or hydroponic pads of various types. Microgreen shoot yield (fresh or dry) results are also in agreement with previously reported ranges $[9,20,21]$.

Microgreen yield varied between the two experiments, with higher fresh shoot weight, macro-, and micronutrient concentrations except for $\mathrm{Cu}$ in the January experiment than February (data not shown). This is likely due to the fluctuating microenvironment conditions in the greenhouse including temperature, relative humidity, and light conditions [26]. Air temperature was set to be $25^{\circ} \mathrm{C}$ in the greenhouse, and was observed to fluctuate within $8{ }^{\circ} \mathrm{C}$ of the setting. Relative humidity ranged from $20 \%$ to $80 \%$, and daily light integral ranged from 3 to $15 \mathrm{~mol} \cdot \mathrm{m}^{-2} \cdot \mathrm{d}^{-1}$ within the experiment duration. Producers could experience similar changes in microgreens between production cycles. Supplemental lighting may help offset such fluctuations.

Fresh microgreen yield was shown to increase with increasing seed sowing rate. However, as seeding rate increases, individual shoot weight decreases and production cost increases since seeds contribute to a significant portion of microgreen production cost $[4,18]$. The sowing rates used in this study was recommended by the seed supplier ranging from $60.5 \mathrm{~g} \cdot \mathrm{m}^{-2}$ in mustard to $189 \mathrm{~g} \mathrm{~m}^{-2}$ in radish (Table 1), equivalent to 17,182 seeds per $\mathrm{m}^{2}$ in radish to 33,611 seeds per $\mathrm{m}^{2}$ in mustard. This sowing rate was generally in agreement with reported ranges of 10,000-40,000 seeds per $\mathrm{m}^{2}$ for microgreens, yet crop-specific optimum sowing rate needs to be determined based on germination percentage, seed weight, and desired shoot density [27]. Besides fresh shoot yield, choice of microgreen species is also affected by appearance, texture, flavor, nutritional values, and phytochemical compositions, with genetic variability between and within taxa for traits of interest $[9,28]$.

Compared to a number of studies investigating the effect of light on minerals and phytochemical concentrations in microgreens $[19,29,30]$, there lacks standardization in cultural practices including fertilization application, or guidelines for microgreen quality $[4,20,24,25]$. One time post-emergent fertigation increased overall shoot height in both experiments, increased overall dry shoot weight and fresh shoot weight in kale and radish in January 2020, and increased various macronutrient concentrations in different microgreens. This is in agreement with our previous results evaluating ten microgreens using a peat-based soilless substrate, when one time post-emergent fertigation with $100 \mathrm{mg} \cdot \mathrm{L}^{-1} \mathrm{~N}$ also increased overall fresh shoot yield, macro-, and micronutrient concentrations in selected microgreen species. Murphy et al. [4] recommended daily fertigation with $150 \mathrm{mg} \cdot \mathrm{L}^{-1} \mathrm{~N}$ for optimal yield in arugula microgreens [18]. In addition to increased yield, large shoot height is a desirable characteristic in assisting with easy harvest of microgreens manually or mechanically [11]. Hydroponic pads generally have lower water holding capacity than peat-based substrate [21], microgreens grown in a hydroponic system may benefit from more frequent fertigation. Slow growing species may require more frequent fertilization than fast growing ones [1]. 
Mineral nutrient profiles varied among microgreens. For example, radish and broccoli had the highest $\mathrm{N}$ concentrations and mustard had the highest $\mathrm{P}$ concentration when grown in three substrate types except for hemp. Mustard also had the lowest $\mathrm{N}$ concentration among tested microgreens when grown with each substrate type. Ranking of most other mineral nutrients between microgreens varied among hydroponic pad types, suggesting substrate type can affect nutrient profile of microgreens, which should be taken into consideration in microgreen production. Analyses of 30 varieties of Brassica microgreens revealed that they are a rich source of mineral nutrients, especially $\mathrm{K}, \mathrm{Mg}$, $\mathrm{Fe}$, and $\mathrm{Zn} \mathrm{[16].} \mathrm{Concentra-}$ tions of macro- and micronutrients in the current study were generally in consistent ranges with previous reports. The five tested microgreens had lower concentrations of $\mathrm{K}, \mathrm{Cu}$, $\mathrm{Fe}$, and $\mathrm{B}$ in this study grown with hydroponic pads than when grown with a peat-based potting mix in our previous study [26]. This agrees with Di Gioia et al. [21] in that peat resulted in higher nitrate, potassium, and sodium concentrations in microgreen rapini than three hydroponic pads including Sure to Grow (made from polyethylene-terephthalate), textile fiber, and jute-kenaf fiber. This may result from the facts that water holding capacity of peat was tested to be higher than hydroponic pads, and that peat itself contains higher mineral nutrient concentrations than the hydroponic pads [21]. Mineral nutrients may also change among harvest stages from microgreen stage 1, microgreen stage 2, baby leaf, to adult stage, with microgreen stages having higher mineral nutrient concentrations than the adult stage [5].

An ideal hydroponic growing substrate should be readily available, relatively inexpensive, and derived from renewable materials [21]. They should also have an adequate ratio between micropores to macropores to have high container capacity and sufficient air filled porosity. The four tested hydroponic pads are all constructed with biodegradable fibers with different physical and chemical characteristics. Hemp mat was superior in producing the highest shoot height, fresh and dry shoot weights in each microgreen species, or overall, in one or two experiments compared with the other three pad types. Hemp also resulted in the lowest $\mathrm{N}$ concentrations among substrate types in each microgreen in both experiments, which could result from the dilution effect of nutrient due to the highest fresh and dry shoot weights in microgreens. Fast growth and high yield of microgreens may benefit from fertilization with a high $\mathrm{N}$ formula, with caution on the balance between nitrate and ammonium forms of $\mathrm{N}$ since some microgreen crops like arugula tends to accumulate more nitrate [20]. However, microgreens generally have lower nitrate concentrations than their mature counterparts $[10,12]$. Compared to the hemp mat, jute, and micro-mats have denser texture, likely contributing to a higher water holding capacity but less air filled porosity, while biostrate is thin and lose moisture easily as observed.

Hydroponic pads constructed with different fiber materials can be sources of certain mineral nutrients [21]. A hemp mat could be a source of $K$ and $B$, resulting in the highest $\mathrm{K}$ (in both experiments) and B concentrations (in January) in each microgreen. Highest micronutrient concentrations were mostly found in the jute or hemp mat if there was significant difference among substrate types, where the higher nutrient concentrations may also result from better shoot growth in these two substrate types. Further analyses of mineral compositions of different hydroponic pads will be required to confirm their effects on microgreen mineral nutrient concentrations.

Food safety concerns including microbial contamination by human pathogens in microgreen production has emerged with the expanding industry [7]. Microbial contamination can be introduced through seeds, growing substrate, equipment, or a lack of hygienic practices by workers [31]. Microgreens are more vulnerable to internalization of bacteria than mature vegetable plants [32]. However, foodborne pathogen outbreaks have not been associated with microgreen consumption as much as in sprouts, where sprouts are generally grown in a dark and moist condition that are more conductive to microbial proliferation [9,33-35]. We did observe mold-like occurrence on hydroponic pads, with higher mold counts on micro-mats and hemp mats, less in our previous study using a peat-based substrate [26]. It was recently reported that microgreens grown in a hydroponic 
system and soil-substitute are both subject to pathogen proliferation with contaminated seeds, with higher microbial population in the hydroponic system [33,36,37]. Effective treatments for seed surface sterilization and antimicrobial action need to be developed for sustainable microgreen production $[9,38]$. Disinfecting practices should also be identified for hydroponic pads since they could be potential source of microbial contamination [21]. Growers should also select reputable supplier for certified microgreen or sprout seeds with high germination quality and potentially lower health risks.

\section{Conclusions}

Microgreens varied in their shoot growth and mineral nutrient concentrations. Hydroponic pads with varying physical and chemical properties affected shoot yield and mineral nutrients of microgreens. Microgreens in the hemp mat showed the highest fresh and dry shoot weight, likely due to the appropriate balance between water holding capacity and air filled porosity. One time post-emergent fertilization increased overall shoot height of tested microgreens in both experiments. Recommendation for fertilization should be adjusted to species/varieties and production conditions.

Supplementary Materials: The following are available online at https:/ /www.mdpi.com/article/10 $.3390 /$ horticulturae7060129/s1, Figure S1: Growth stage of five microgreens at harvest including 'Waltham' broccoli (A), 'Red Acre' cabbage (B), 'Red Russian' kale (C), 'Red Garnet' mustard (D), and Rambo radish (E).

Author Contributions: Conceptualization, T.L.; investigation, G.T.L., J.D.A., M.H.J. and T.L.; writing —original draft preparation, T.L.; writing — review and editing, G.B. and J.D.A.; funding acquisition, T.L. and G.B. All authors have read and agreed to the published version of the manuscript.

Funding: This work was supported by the United States Department of Agriculture (USDA) Mississippi Department of Agriculture and Commerce Specialty Crop Block Grant Program (G00003962) and the United States Department of Agriculture (USDA) National Institute of Food and Agriculture Hatch Project MIS-112040. Mention of a trademark, proprietary product, or vendor, does not constitute a guarantee or warranty of the product by Mississippi State University or the USDA and does not imply its approval to the exclusion of other products or vendors that also may be suitable.

Institutional Review Board Statement: Not applicable.

Conflicts of Interest: The authors declare no conflict of interest.

\section{References}

1. Treadwell, D.D.; Hochmuth, R.; Landrum, L.; Laughlin, W. Microgreens: A New Specialty Crop. Univ. Florida IFAS Ext. 2020, HS1164. Available online: https:/ /edis.ifas.ufl.edu/pdffiles/HS/HS116400.pdf (accessed on 7 December 2020).

2. Gerovac, J.R.; Craver, J.K.; Boldt, J.K.; Lopez, R.G. Light intensity and quality from sole-source light emitting diodes impact growth, morphology, and nutrient content of Brassica microgreens. HortScience 2016, 51, 497-503. [CrossRef]

3. Bachman, G.R. Growing Microgreens for the Mississippi Gardener; Mississippi State University Extension Service: Starkville, MS, USA, 2014; p. 2857.

4. Murphy, C.J.; Llort, K.F.; Pill, W.G. Factors affecting the growth of microgreen table beet. Int. J. Veg. Sci. 2010, 16, $253-266$. [CrossRef]

5. Waterland, N.L.; Moon, Y.; Tou, J.C.; Kim, M.J.; Pena-Yewtukhiw, E.M.; Park, S. Mineral content differs among microgreen, baby leaf, and adult stages in three cultivars of kale. HortScience 2017, 52, 566-571. [CrossRef]

6. Xiao, Z.; Rausch, S.R.; Luo, Y.; Sun, J.; Yu, L.; Wang, Q.; Chen, P.; Yu, L.; Stommel, J.R. Microgreens of Brassicaceae: Genetic diversity of phytochemical concentrations and antioxidant capacity. LWT Food Sci. Technol. 2019, 101, 731-737. [CrossRef]

7. Turner, E.R.; Luo, Y.; Buchanan, R.L. Microgreen nutrition, food safety, and shelf life: A review. J. Food Sci. 2020, 85, 870-882. [CrossRef]

8. Bachman, G.R. Microgreens Varieties for the Mississippi Gardener; Mississippi State University Extension Service: Starkville, MS, USA, 2015; p. 2884.

9. Kyriacou, M.C.; Rouphael, Y.; Di Gioia, F.; Kyratzis, A.; Serio, F.; Renna, M.; De Pascale, S.; Santamaria, P. Micro-scale vegetable production and the rise of microgreens. Trends Food Sci. Technol. 2016, 57, 103-115. [CrossRef]

10. Pinto, E.; Almeida, A.A.; Aguiar, A.A.; Ferreira, I.M.P.L.V.O. Comparison between the mineral profile and nitrate content of microgreens and mature lettuces. J. Food Compos. Anal. 2015, 37, 38-43. [CrossRef] 
11. Ying, Q.; Kong, Y.; Zheng, Y. Growth and appearance quality of four microgreen species under light-emitting diode lights with different spectral combinations. HortScience 2020, 55, 1399-1405. [CrossRef]

12. Xiao, Z.; Lester, G.F.; Luo, Y.; Wang, Q. Assessment of vitamin and carotenoid concentrations of emerging food products: Edible microgreens. J. Agric. Food Chem. 2012, 60, 7644-7651. [CrossRef] [PubMed]

13. Kyriacou, M.C.; El-Nakhel, C.; Graziani, G.; Pannico, A.; Soteriou, G.A.; Giordano, M.; Ritieni, A.; De Pascale, S.; Rouphael, Y. Functional quality in novel food sources: Genotypic variation in the nutritive and phytochemical compostion of thirteen microgreens species. Food Chem. 2019, 277, 107-118. [CrossRef]

14. Nadihira, S.; Ramar, A.; Jegadeeswari, V.; Srinivasan, S. Microgreen production in herbal spices. J. Pharmacogn. Phytochem. 2021, 10, 168-170.

15. Johnson, S.A.; Prenni, J.E.; Heuberger, A.L.; Isweiri, H.; Chaparro, J.M.; Newman, S.E.; Uchanski, M.E.; Omerigic, H.M.; Michell, K.A.; Bunning, M.; et al. Comprehensive evaluation of metabolites and minerals in 6 microgreen species and the influence of maturity. Curr. Dev. Nutr. 2021, 5, nzaa180. [CrossRef] [PubMed]

16. Xiao, Z.; Codling, E.E.; Luo, Y.; Nou, X.; Lester, G.E.; Wang, Q. Microgreens of Brassicaceae: Mineral composition and content of 30 varieties. J. Food Compos. Anal. 2016, 49, 87-93. [CrossRef]

17. Puccinelli, M.; Malorgio, F.; Rosellini, I.; Pezzarossa, B. Production of selenium-biofortified microgreens from selenium-enriched seeds of basil. J. Sci. Food Agric. 2019, 99, 5601-5605. [CrossRef] [PubMed]

18. Murphy, C.J.; Pill, W.G. Cultural practices to speed the growth of microgreen arugula (roquette; Eruca vesicaria subsp. sativa). J. Hort. Sci. Biotechnol. 2010, 85, 171-176. [CrossRef]

19. Brazaitytè, A.; Vaštakaite, V.; Viršilè, A.; Jankauskienè, J.; Samuolienè, G.; Sakalauskienè, S.; Novičkovas, A.; Miliauskienė, J.; Duchovskis, P. Changes in mineral element content of microgreens cultivated under different lighting conditions in a greenhouse. Acta Hort. 2018, 1227, 507-516. [CrossRef]

20. Bulgari, R.; Baldi, A.; Ferrante, A.; Lenzi, A. Yield and quality of basil, Swiss chard, and rocket microgreens grown in a hydroponic system. N. Z. J. Crop Hort. Sci. 2017, 45, 119-129. [CrossRef]

21. Di Gioia, F.; De Bellis, P.; Minini, C.; Santamaria, P.; Serio, F. Physicochemical, agronomical and microbiological evaluation of alternative growing media for the production of rapini (Brassica rapa L.) microgreens. J. Sci. Food Agric. 2017, 97, 1212-1219. [CrossRef]

22. Rajan, P.; Lada, R.R.; MacDonald, M.T. Advancement in indoor vertical farming for microgreen production. Am. J. Plant Sci. 2019, 10, 1397-1408. [CrossRef]

23. Kou, L.P.; Luo, Y.G.; Yang, T.B.; Xiao, Z.L.; Turner, E.R.; Lester, G.E.; Wang, Q.; Camp, M.J. Postharvest biology, quality and shelf life of buckwheat microgreens. LWT Food Sci. Technol. 2013, 51, 73-78. [CrossRef]

24. Kou, L.; Yang, T.; Luo, Y.; Liu, X.; Huang, L.; Codling, E. Pre-harvest calcium application increases biomass and delays senescence of broccoli microgreens. Postharvest Biol. Technol. 2014, 87, 70-78. [CrossRef]

25. Sun, J.; Kou, L.; Geng, P.; Huang, H.; Yang, T.; Luo, Y.; Chen, P. Metabolomic assessment reveals an elevated level of glucosinolate content in $\mathrm{CaCl}_{2}$ treated broccoli microgreens. J. Agric. Food Chem. 2015, 63, 1863-1868. [CrossRef]

26. Li, T.; Lalk, G.T.; Bi, G. Fertilization and Pre-sowing seed soaking affect yield and mineral nutrients of ten microgreen species. Horticulturae 2021, 7, 14. [CrossRef]

27. Di Gioia, F.; Santamaria, P. Microgreens: Novel, Fresh and Functional Food to Explore All the Value of Biodiversity; ECO-Logica: Bari, Italy, 2015.

28. Xiao, Z.; Lester, G.E.; Park, E.; Saftner, R.A.; Luo, Y.; Wang, Q. Evaluation and correlation of sensory attributes and chemical compositions of emerging fresh produce: Microgreens. Postharvest Biol. Technol. 2015, 110, 140-148. [CrossRef]

29. Alrifai, O.; Hao, X.; Marcone, M.F.; Tsao, R. Current review of the modulatory effects of LED lights on photosynthesis of secondary metabolites and future perspectives of microgreen vegetables. J. Agric. Food Chem. 2019, 67, 6075-6090. [CrossRef]

30. Kopsell, D.A.; Sams, C.E. Increases in shoot tissue pigments, glucosinates, and mineral elements in sprouting broccoli after exposure to short-duration blue light from light emitting diodes. J. Am. Soc. Hort. Sci. 2013, 138, 31-37. [CrossRef]

31. Yang, Y.; Meier, F.; Lo, J.A.; Yuan, W.; Sze, V.L.P.; Chung, H.L.; Yuk, H.G. Overview of recent events in the microbiological safety of sprouts and new intervention technologies. Compr. Rev. Food Sci. Food Saf. 2013, 12, 265-280. [CrossRef]

32. Warriner, K.; Ibrahim, F.; Dickinson, M.; Wright, C.; Waites, W.M. Internalization of human pathogens within growing salad vegetables. Biotechnol. Genet. Eng. Rev. 2003, 20, 117-136. [CrossRef] [PubMed]

33. Xiao, Z.; Bauchan, G.; Nichols-Russell, L.; Luo, Y.; Wang, Q.; Nou, X. Proliferation of Escherichia coli O157:H7 in soil-substitute and hydroponic microgreen production systems. J. Food Prot. 2015, 78, 1785-1790. [CrossRef] [PubMed]

34. Xiao, Z.; Nou, X.; Luo, Y.; Wang, Q. Comparison of the growth of Escherichia coli O157: H7 and O104: H4 during sprouting and microgreen production from contaminated radish seeds. Food Microbiol. 2014, 44, 60-63. [CrossRef]

35. Ebert, A. Spouts, microgreens, and edible flowers: The potential for high value specialty produce in Asia. In High Value Vegetables in Southeast Asia: Production, Supply, and Demand, Proceedings of the SEAVEG 2012, Chiang Mai, Thailand, 24-26 January 2012; Asian Vegetable Research and Development Center: Tainan, Taiwan, 2013; pp. 216-227.

36. Reed, E.; Ferreira, C.M.; Bell, R.; Brown, E.W.; Zheng, J. Plant-microbe and abiotic factors influencing salmonella survival and growth on alfalfa sprouts and Swiss chard microgreens. Appl. Environ. Microbiol. 2018, 84, e02814-17. [CrossRef] [PubMed] 
37. Wright, K.M.; Holden, N.J. Quantification and colonisation dynamics of Escherichia coli O157:H7 inoculation of microgreens species and plant growth substrates. Int. J. Food Microbiol. 2018, 273, 1-10. [CrossRef] [PubMed]

38. Ding, H.; Fu, T.J.; Smith, M.A. Microbial contamination in sprouts: How effective is seed disinfection treatment? J. Food Sci. 2013, 78, 495-501. [CrossRef] [PubMed] 